\title{
Chapter 7 \\ Laser Interactions for the Synthesis and In Situ Diagnostics of Nanomaterials
}

\author{
David B. Geohegan, Alex A. Puretzky, Mina Yoon, Gyula Eres, Chris \\ Rouleau, Kai Xiao, Jeremy Jackson, Jason Readle, Murari Regmi, \\ Norbert Thonnard, Gerd Duscher, Matt Chisholm and Karren More
}

\begin{abstract}
Laser interactions have traditionally been at the center of nanomaterials science, providing highly nonequilibrium growth conditions to enable the synthesis of novel new nanoparticles, nanotubes, and nanowires with metastable phases. Simultaneously, lasers provide unique opportunities for the remote characterization of nanomaterial size, structure, and composition through tunable laser spectroscopy, scattering, and imaging. Pulsed lasers offer the opportunity, therefore, to supply the required energy and excitation to both control and understand the growth processes of nanomaterials, providing valuable views of the typically nonequilibrium growth kinetics and intermediates involved. Here we illustrate the key challenges and progress in laser interactions for the synthesis and in situ diagnostics of nanomaterials through recent examples involving primarily carbon nanomaterials, including the pulsed growth of carbon nanotubes and graphene.
\end{abstract}

\subsection{Introduction}

The special properties of nanomaterials originate from size-dependent atomic configurations that often result in a departure of electronic band structure, chemical reactivity, and physical properties from their bulk counterparts [1].

\footnotetext{
D. B. Geohegan $(\bowtie) \cdot$ A. A. Puretzky · M. Yoon · C. Rouleau · K. Xiao · J. Jackson Center for Nanophase Materials Sciences, Oak Ridge National Laboratory, Oak Ridge, TN 37831, USA e-mail: geohegandb@ornl.gov

G. Eres · J. Jackson · J. Readle · M. Regmi · M. Chisholm · K. More Materials Science and Technology Division, Oak Ridge National Laboratory, Oak Ridge, TN 37831, USA

N. Thonnard · G. Duscher

Department of Materials Science and Engineering, University of Tennessee, Knoxville, TN 37996, USA
} 


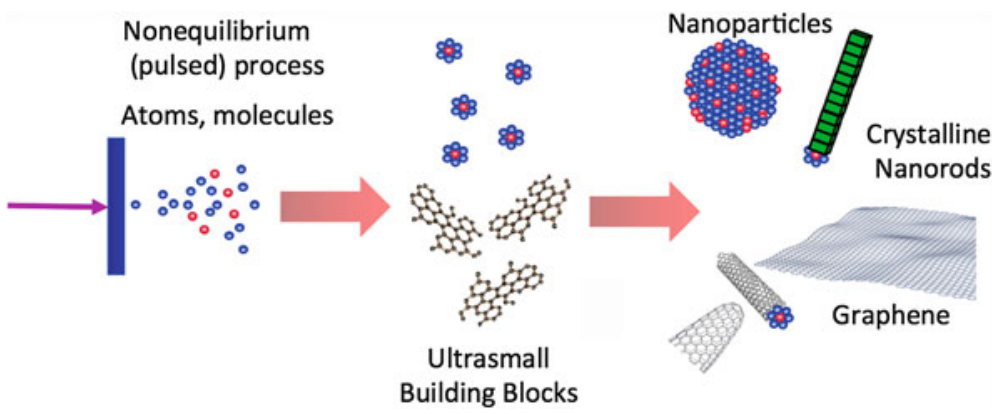

Fig. 7.1 Schematic of the synthesis of nanomaterials from nonequilibrium, pulsed laser-induced process such as laser vaporization of a target into atoms and molecules. A great variety of nanoparticles, crystalline nanorods, and polymorphs (e.g., for carbon, nanohorns, nanotubes, graphene, etc.) are energetically possible, both with and without catalyst-assistance, depending on the nucleation and growth of intermediate, ultrasmall building blocks and subsequent growth kinetics

Under equilibrium synthesis conditions, thermodynamic energy barriers severely constrain the number of configurations and structures that can occur [2]. However, numerous metastable configurations can emerge during synthesis as a result of competition between thermodynamic and kinetic pathways [3]. Non-equilibrium growth methods can overcome the thermodynamic energy barriers for metastable nanostructure formation, and are important tools for materials discovery.

Laser interactions with materials provide remarkably versatile nonequilbrium conditions for nanomaterials synthesis. Laser vaporization of solid targets, for example, typically results in stoichiometric material removal, plasma formation $\left(T_{e} \sim 1-10 \mathrm{eV}\right)$, and translational kinetic energies up to $\sim 100 \mathrm{eV}[4,5]$. Spatial confinement of these reactants using a background gas can provide the time, temperature, and fluxes of reactants necessary for the formation of nanostructures with metastable phases. In carbon nanomaterials, as shown in Fig. 7.1 for example, a great variety of nanostructures may emerge depending upon the nucleation and growth pathways induced by this spatial confinement. Various allotropes of carbon can be formed depending on the growth times and temperatures provided by the spatial confinement, including fullerenes, [6] carbon nanohorns [7, 8], nanodiamond [9], graphene [10], and carbon nanotubes [11]. For other materials, a great variety of nanoparticles, crystalline nanorods, nanotubes, planar materials, and curved structures can similarly be obtained under such nonequilibrium growth conditions [12]. Metal catalysts introduced into the reactant mixture can greatly alter the product distribution by lowering reaction barriers in chemical reactions. As a result, metal catalyst-assisted chemical vapor deposition is often explored to duplicate a subset of the nanomaterials (such as single wall nanotubes (SWNTs), silicon nanowires (SiNWs), etc.) discovered by laser vaporization.

However, as indicated in Fig. 7.1, understanding and controlling the growth processes that determine the atomic structure of different forms of nanostructures 
remains a fundamental grand challenge [13] for nanoscience. Can laser interactions reveal the nuclei and ultrasmall building blocks that form the basis for the growth and assembly of larger nanostructures? Can pulsed laser interactions reveal the timescales for their formation? Answering these questions requires real-time diagnostics of the growth environment and nanostructure formation. Through time- and spatially-resolved plasma spectroscopy, the concentrations of atomic and molecular reactants as well as plasma temperatures can be remotely determined in situ (see [4, 5]). Moreover, through optical spectroscopy, the size and structure of many nanostructures can be remotely determined, as well as their evolution in size and concentration. Therefore, valuable kinetics information can be inferred which can be used to model nucleation and growth kinetics, and from which the presence of reactive intermediates may be inferred. A major challenge remaining for laser interactions with materials is the development of laser-based, real-time diagnostics that can identify the ultrasmall building blocks and reactive intermediates in situ.

In this chapter, current challenges in laser interactions with materials for both synthesis and in situ diagnostics of nanomaterials growth processes will be illustrated with an emphasis on carbon nanomaterials. The evidence for "ultrasmall building blocks" in nanomaterials growth will be examined through a review of both high- and low-temperature nanomaterials synthesis processes, including laser vaporization and chemical vapor deposition.

\subsection{Cluster and Nanoparticle Growth in Pulsed Laser Vaporization}

Pulsed laser vaporization (PLV) of targets using nanosecond lasers typically results in an energetic plasma plume that can penetrate moderate pressure background gases with sufficient deposition rate for the pulsed laser deposition (PLD) of thin films (pressures typically $<300$ mTorr for reasonable distances of 5-10 cm). As indicated in Fig. 7.2a, atoms and molecules comprise the principal components of the ablation plume arriving at the substrate in vacuum. However, as the background gas pressure is raised, the plasma plume is slowed and confined, leading to the condensation of clusters and nanoparticles as shown in Fig. 7.2b. Under these conditions, the film morphology can completely change.

Measurements of the ion flux penetrating through different background gas pressures reveals that material is scattered from the plume in accordance with Beer's law with an elastic scattering cross section $\sim 1 \times 10^{-16} \mathrm{~cm}^{2}$, with material from the initial shifted center of mass Maxwell-Boltzmann distribution disappearing exponentially with background gas pressure or distance [14]. At particular distances and pressures, some fraction of the initial 'vacuum' distribution of ions can still be detected while a second, delayed component of material arrives much later in time [15]. This situation is illustrated in Fig. 7.2d for the case of ZnTe target ablation into background nitrogen gas [16]. At a distance of $10 \mathrm{~cm}$ from the 


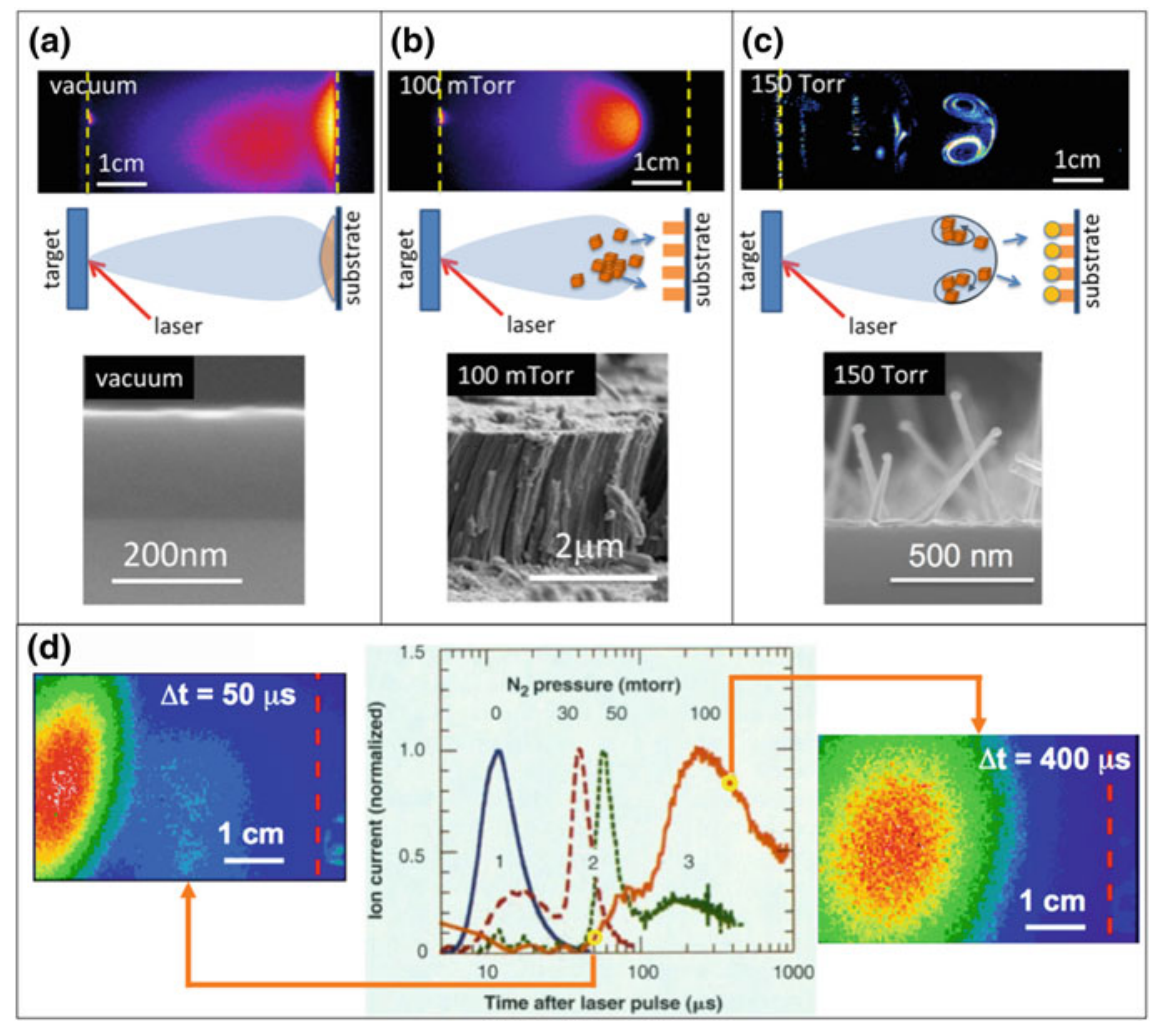

Fig. 7.2 a Gated-ICCD images of laser plasma plumes for PLD in vacuum, b 100 mTorr, and swirling clouds of aggregated nanoparticles at $\mathbf{c} 150$ Torr (Rayleigh scattered light from a second laser sheet). Corresponding schematics show that PLD growth on a substrate changes results from atoms and molecules in vacuum, and an increasing fraction of clusters and nanoparticles in higher pressure background gases. The resulting synthesized structures can be varied from a smooth epitaxial films (e.g. $\mathrm{TiO}_{2}$ ), to b columnar nanoparticle assembles (e.g. $\mathrm{TiO}_{2}$ ), to c crystalline nanorods (e.g. $\mathrm{SnO}_{2}$ on $\mathrm{Au} / \mathrm{Si}$ ). d Ion probe current pulses (center panel) measured at $\mathrm{d}=10 \mathrm{~cm}$ from a ZnTe target in background pressures ranging from 0 to $100 \mathrm{mTorr}_{2}$ show corresponding evolution of 3 plume components: (1) atoms and molecules, (2) atoms and molecules + clusters, (3) clusters and nanoparticles. Corresponding images at two times in $100 \mathrm{mTorr}$ show the position of the third component of the plume at $\Delta \mathrm{t}=50 \mu \mathrm{s}$ (left) and at $\Delta \mathrm{t}=400 \mu \mathrm{s}$ (right) after laser ablation relative to $\mathrm{d}=10 \mathrm{~cm}$ (red dashed line) [(d) from 16]

target, the initial vacuum distribution of ions arriving to the substrate is labeled "1", while the time-dependence of ions arriving through 30 mTorr $\mathrm{N}_{2}$ shows two components, a fast component labeled " 1 " and a slowed component labeled " 2 ". This "plume-splitting" phenomenon is a special circumstance which is difficult to detect using plume imaging alone because the fast 'vacuum' component at the front of the plume represents that material which has penetrated the background gas without collisions. This fast component is far less bright in comparison to the delayed second component, where collision-induced fluorescence resulting from 
slowed atoms and molecules condensing into a 'shock front' can become very bright. Despite the fact that this highly visible component of the plume is often used to track the apparent propagation of the plume propagation (using, shock, drag, or other models which assume hydrodynamic propagation) the successful description of plume splitting which takes into account both components of the plume involves a scattering model formalism [17]. Plume splitting is a general phenomenon which has been successfully described using a single elastic scattering cross section for both atoms propagating into lighter, or heavier, gases [18].

At longer distances, this second component can be seen to split into yet another, third component labeled " 3 " in Fig. 7.2d. This component corresponds to larger nanoparticles and nanoparticle aggregates which retain some charge from the plasma. Photoluminescence from neutral nanoparticles and their aggregates can be induced by a second, delayed laser pulse as shown in the two images in Fig. 7.2d [19]. Thus, at low pressures and different distances from the target, mixtures of fast atoms and molecules with clusters can be replaced with a flux composed of nearly entirely nanoparticles and their aggregates. We label this nanoparticle assisted PLD (NA-PLD) in Fig. 7.2b.

At higher pressures, the plume condenses rapidly into nanoparticles, which aggregate and become trapped in sharp vortex rings [20], a cross-section of which is shown in Fig. 7.2c. With metal catalyst-doped targets, a great variety of semiconductor nanowires, nanorods, and nanotubes can be grown while this material is suspended at high temperatures within a tube furnace, although the process is typically referred to as a VLS, or vapor-liquid-solid process [12]. Evidence suggests that small clusters and ultrasmall nanoparticles dominate the "vapor" in such catalyst-assisted growth processes. This can be explicitly demonstrated, as indicated in Fig. 7.2c, by directing preformed nanoparticles as the feedstock for the growth of nanowires on substrates. In Fig. 7.2c $\mathrm{SnO}_{2}$ nanowires are shown growing from $\mathrm{Au}$ catalyst nanoparticles at their tips, demonstrating that the aggregated nanoparticles in 150 Torr background $\mathrm{Ar}$ following $\mathrm{SnO}_{2}$ target ablation can serve as feedstock to nucleate and grow nanowires on a substrate within a tube furnace in a nanoparticle-assisted chemical vapor deposition (CVD) process. Similarly, imaging of $\mathrm{ZnO}$ ablation at high pressures was used to demonstrate that crystalline $\mathrm{ZnO}$ nanorods can be grown by nanoparticle-assisted PLD without the use of any metal catalyst layer [21]. Hence, the role and dynamics of small nanoparticles as "building blocks" in the assembly of crystalline thin films and nanostructures remains a key question for in situ diagnostic experiments.

\subsection{Characterization and Modeling of Ultrasmall Nanoparticle "Building Blocks"}

What determines the size, shape, stoichiometry, and crystallinity of nanostructures formed within the laser plume? Spatial confinement of the plume, time, and temperature determine the synthesis conditions for the thermalization and 
condensation of atoms and molecules into clusters and nanoparticles. This has been modeled for the idealized isentropic expansion of a plume into vacuum [22], however the dynamics of plume expansion into background gases plays a major role in the adjustment of these parameters. As described above, the standard hydrodynamic models for plume expansion do not accurately describe low-pressure plume expansions where a scattering formalism is more appropriate.

Only recently, with the advent of aberration-corrected atomic-resolution transmission electron microscopy, have such views of the atomic structure of ultrasmall nanoparticles (UNPs, diameters $<3 \mathrm{~nm}$ ) become available. Figure 7.3a, b show high resolution TEM and atomic-resolution Z-contrast scanning TEM images of $\mathrm{TiO}_{2}$ nanoparticle aggregates collected on TEM grids under ablation of a $\mathrm{TiO}_{2}$ target into low-pressure, room temperature background argon. Such HRTEM and EELS data of $\mathrm{TiO}_{2}$ UNPs show that the individual nanoparticles are typically crystalline (see Fig. 7.3a and b) with structures that do not match those of known bulk phases. It is quite likely that such nanoparticles, which are formed under highly nonequilibrium conditions, are energetically metastable. In addition, the high fraction of surface atoms leads to undercoordinated bonding and energetically active surfaces. The chemistry induced by such surfaces may facilitate the oriented attachment of the particles [23, 24].

Identifying and classifying unknown phases of ultrasmall metastable nanoparticles is extremely challenging, and requires new computational approaches that are capable of rapidly surveying the energy landscape of a wide range of nanoparticle morphologies and structures. However, most atomistic studies of ultrasmall nanoparticle structure have assumed a known bulk phase as a starting point, which is then permitted to undergo relaxation at different temperatures [25-28]. Even with this limited approach, first principles modeling is currently computationally tractable only for nanoparticles with sizes $<3 \mathrm{~nm}$. Hybrid approaches combining force-field potentials and first principles calculations are being developed to rapidly survey configuration space to identify unknown structures.

Figure $7.3 \mathrm{c}$ and $\mathrm{d}$ show three minimum-energy nanoparticle structures that emerged from numerical simulations that began with $1 \mathrm{~nm}$ nanoparticles sculpted from bulk anatase and rutile phases of $\mathrm{TiO}_{2}$, respectively. The structural stability increases from left to right. Here a hybrid method using Monte Carlo (MC) simulations and conjugate gradient (CG) optimization has been used. The total energy is based on the Matsui-Akaogi force-field potential [29] for its relative accuracy as compared to the ab initio methods. The technique's accuracy was verified against previously published results on small $\left(\mathrm{TiO}_{2}\right)_{n}$ systems $(\mathrm{n}=1,2$, and 3 ) based on ab initio approaches (quantum chemistry and density functional theory) [30].

The results clearly demonstrate that the structural properties and the thermodynamic stabilities of ultrasmall nanoparticles can be very different to their bulk counterparts. For example, localized melting of 2-nm anatase is found to start at just $\mathrm{T}=500 \mathrm{~K}$ and persists until $\sim \mathrm{T}=1,000 \mathrm{~K}$, as compared to the bulk melting point of $\sim 2,150 \mathrm{~K}$, with regions of the nanoparticle starting first near the 

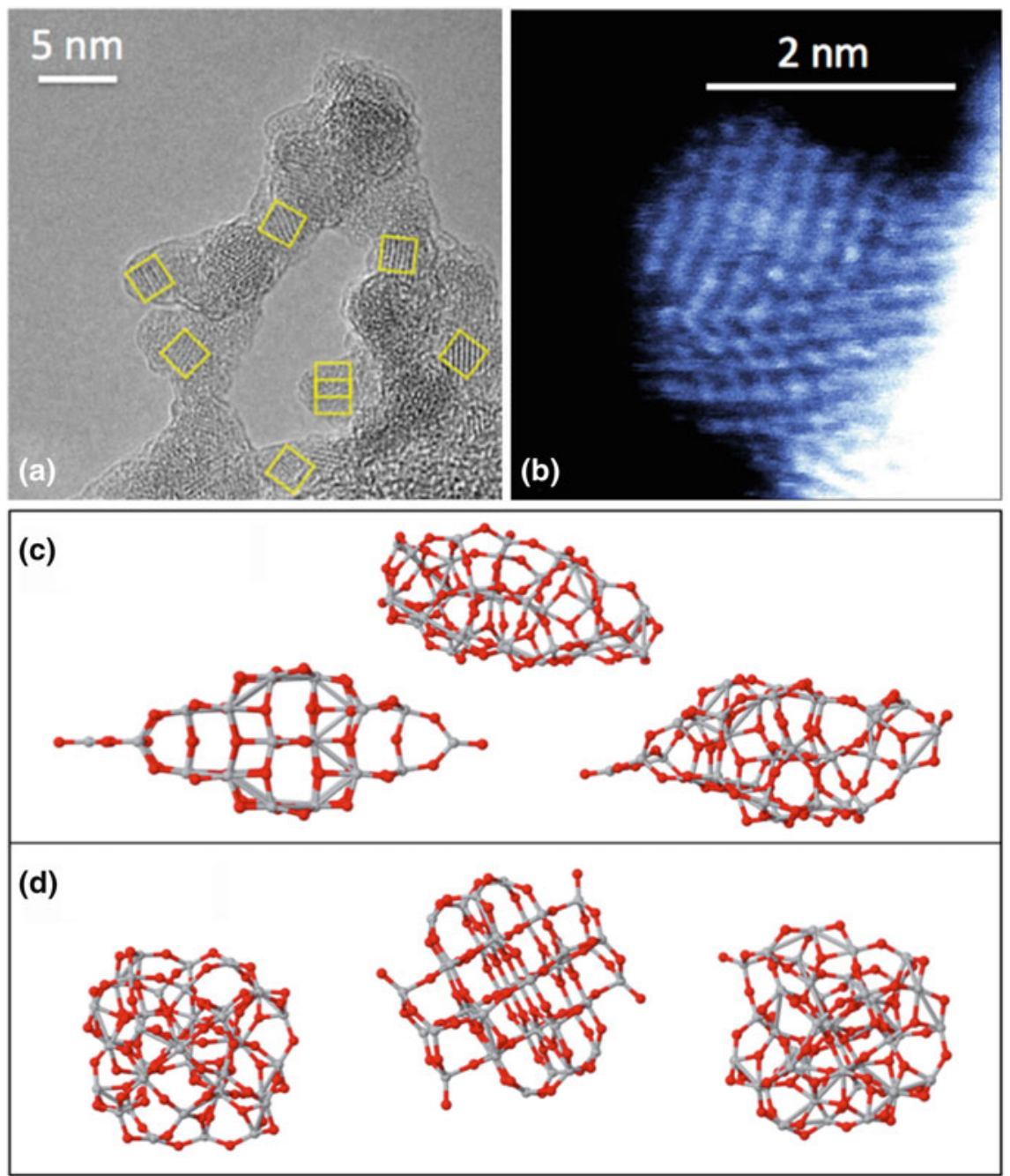

Fig. 7.3 a Aggregated ultrasmall nanoparticles of titanium dioxide formed by $\mathrm{PLV}$ of $\mathrm{TiO}_{2}$ into $100 \mathrm{mTorr}$ of $\mathrm{O}_{2}$. Yellow boxes indicate $2-3 \mathrm{~nm}$ crystalline domains. $100 \mathrm{mTorr}$. b AR-Z-STEM image of a TiOx nanocrystal of an unknown phase. c, d Examples of several local minima theoretically predicted for 1-nm $\mathbf{c}$ anatase and $\mathbf{d}$ rutile nanocrystals of $\mathrm{TiO}_{2}$ which have been allowed to relax into metastable nanophases

surface, then the tips. Such calculations provide a qualitative understanding of how UNPs may serve as "building blocks" and integrate into larger nanoparticles or crystalline nanorods under conditions found in Fig. 7.2 for pulsed laser vaporization, NA-PLD, and nanoparticle assisted-CVD (NA-CVD). 


\subsection{Carbon Nanostructure Synthesis in Laser Vaporization}

\subsubsection{Fullerenes}

Carbon exhibits a remarkable ability to self-assemble into a variety of novel nanostructures within the highly non-equilibrium conditions of laser vaporization plumes. Fullerenes are the early example of carbon clusters with magic numbers that emerged from laser ablation of carbon and condensation in a background gas [31] within specially constructed nozzle sources [32]. Theory and modeling have shown that synthesis temperatures of $\sim 3,000 \mathrm{~K}$ are required to induce the curvature necessary for the formation of fullerenes and other curved carbon nanostructures, while synthesis temperatures of $\sim 1,000-2,000 \mathrm{~K}$ produce flat carbon chain structures and sheets [33]. Pentagons necessary for fullerene curvature can be viewed as local graphene defects, each costing $\sim 45 \mathrm{kcal} / \mathrm{mol}$ energy [34]. However, while the soccer-ball-shaped $\mathrm{C}_{60}$ molecule is relatively easy to produce in the highly non-equilibrium conditions provided by laser ablation or electric arc vaporization of pure carbon rods, for many years computer simulation of the formation process failed to produce the structure of the stable fullerenes. For many years, all of the many reaction pathways and models of fullerene formation were based upon intermediate structures that were in thermodynamic equilibrium. However hot carbon plasmas are far from equilibrium, and such equilibrium models could not describe how such perfect, regular structures like $\mathrm{C}_{60}$ could emerge in such numbers and with such consistency. (See [35] for a good review.)

More recently, Irle et al., considered the nonequilibrium assembly of $\mathrm{C}_{60}$ in hot carbon vapor, proposing a dynamic fullerene self-assemby mechanism wherein a great variety of superfullerenes are formed and then whittled down by the elimination of $\mathrm{C}_{2}$ molecules in a pathway termed the "shrinking hot giant road" [35]. Quantum chemical molecular dynamics (QM/MD) simulations of hot carbon vapor revealed that linear, sp-hybridized carbon polyyne chains nucleate and then assemble into rings, which then condense into curved carbon bowls, and finally, closed irregular giant fullerenes. Following this "size-up" formation of giant fullerenes, the model indicates that the high vibrational temperatures lead to irreversible pop-out processes of $\mathrm{C}_{2}$ molecules or falloff of the weakly-bonded linear antennae of carbon due to violent wagging and stretch vibrations, thereby reducing the size and shape of the molecules toward ever-more circular and stable molecules with fewer dangling bonds and $\mathrm{sp}^{2}$-bonded networks with less strain. Thus, the emergence of stable carbon nanostructures from such a chaotic, nonequilibrium environment was modeled to result from the formation and selfassembly of intermediates into a large variety of products, followed by the ejection of primarily $\mathrm{C}_{2}$ in an annealing period, toward more thermodynamically stable structures [35]. 
Of course, computational cost require that QM/MD simulations are performed at artificially high temperatures for very short (typically picoseconds to nanoseconds) periods, whereas laser plasmas persist for times of microseconds to milliseconds. What are the actual times of formation of carbon nanostructures in the high-temperature laser plasma plumes? To answer this question, time-resolved in situ diagnostics have been employed and their application to understand the catalyst-assisted synthesis of single-wall carbon nanotubes and the catalyst-free assembly of single-wall carbon nanohorns will be summarized below. Additional details have been summarized in other reviews [36].

\subsubsection{Single-Wall Carbon Nanotubes}

Figure 7.4 summarizes key aspects of the laser vaporization growth of single-wall carbon nanotubes (SWNTs) and time-resolved diagnostic experiments. First, in Fig. 7.4a a normal photograph taken within the $1,050{ }^{\circ} \mathrm{C}$ tube furnace shows the glowing circular $1.0^{\prime \prime}$ carbon (with $1 \mathrm{wt}$. \% each $\mathrm{Ni}, \mathrm{Co}$ ) target and many vortex rings of laser vaporized material floating within the gently-flowing Ar gas, silhouetted against the black background. Each vortex ring results from confinement of the ejecta from one laser pulse (laser running at $10 \mathrm{~Hz}$ in these experiments). Confining the reactants into such small volumes for sufficient time for synthesis is crucial for the self assembly of carbon chains and clusters as noted in the modeling simulations above, and also for the catalyst-assisted growth of extended structures like SWNTs, which can reach microns lengths during the 10-s of time these rings remain within the hot growth zone of the furnace.

Time-resolved, in situ imaging and spectroscopy of laser vaporization plumes, schematically illustrated in Fig. 7.4b, utilize a time-delayed sheet beam from a second laser to induce laser-induced fluorescence from atomic or molecular species (e.g., ground state Co atoms in Fig. 7.4c), laser induced blackbody emission from clusters (e.g., C clusters in Fig. 7.4c), or Rayleigh scattering from aggregated clusters (e.g., the vortex rings in Fig. 7.4g) [37, 38]. A time-delayed, gated, intensified CCD-array detector with variable gate $(>3 \mathrm{~ns})$ is typically used to capture images or optical spectra during, or at a well-defined delay with respect to, the probe laser pulse. For example, in Fig. 7.4c, images taken through different colored filters at $\Delta \mathrm{t}=1.0$, and $2.0 \mathrm{~ms}$ after the initial laser (vaporizing) pulse revealed that molecular carbon disappeared by $\Delta \mathrm{t}=1.0 \mathrm{~ms}$ and blackbody emission from carbon clusters appeared. Within another millisecond, the vortex ring trapping aggregated carbon clusters was well established. However, during the same time period, ground state atomic Co was reaching its maximum population at $\Delta \mathrm{t}=1.0 \mathrm{~ms}$ (following relaxation of the hot laser plasma, not shown) and just began to condense during the next millisecond. These measurements revealed that carbon condenses into clusters first, relatively late in time and at extended distances from the target, followed by the condensation of the metal atoms. Thus, the raw feedstock for SWNT growth was condensed phase clusters of carbon and 


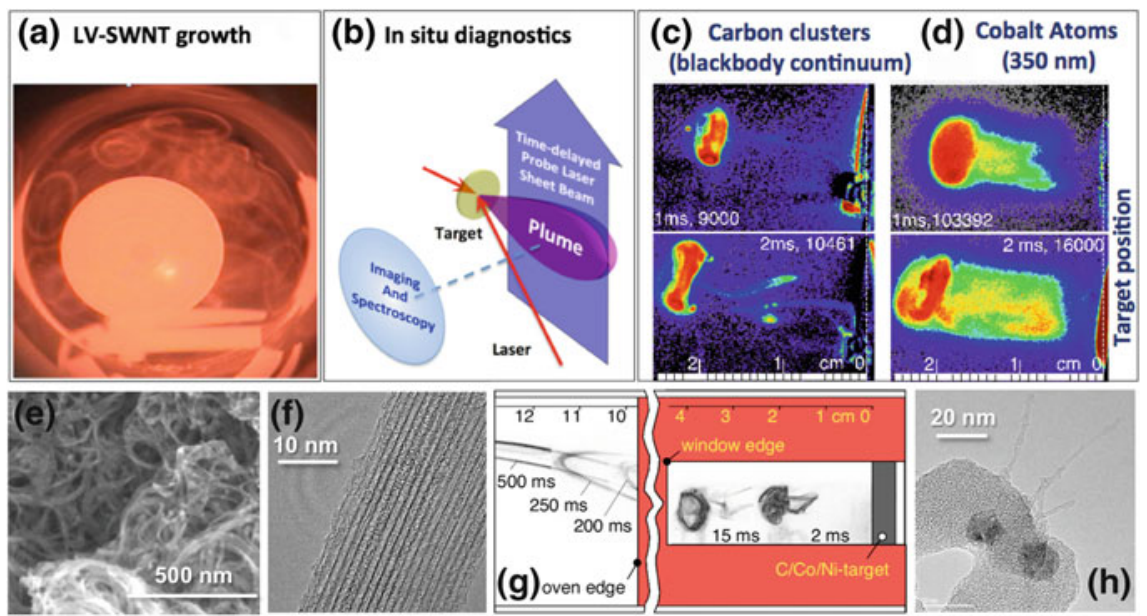

Fig. 7.4 Summary of time-resolved, in situ diagnostic measurements of the laser vaporization growth of SWNTs. a Digital photograph looking down a $1,050{ }^{\circ} \mathrm{C}\left(2.0^{\prime \prime}\right)$ tube furnace along the laser beam direction, showing a $1.0^{\prime \prime} \mathrm{C} / \mathrm{Ni} / \mathrm{Co}$ target, focused $\mathrm{Nd}: \mathrm{YAG}$ laser spot, and many vortex rings wherein the ejecta within each laser pulse become trapped in the 500 Torr Ar background pressure. b Schematic of the typical in situ diagnostic approach for imaging and spectroscopy, including the option for a second, time-delayed laser sheet beam to probe the plume. c, $\mathbf{d}$ Filtered, gated ICCD images of the expanding laser plume from the target at $\Delta \mathrm{t}=1$, $2 \mathrm{~ms}$. Aggregated carbon clusters are observable in vortex dynamics by laser-induced blackbody continuum radiation, while laser-induced $\left(\lambda_{\mathrm{ex}}=308 \mathrm{~nm}\right)$ fluorescence at $350 \mathrm{~nm}$ is used to observe the ground-state Co atoms at the same times. e SEM and $\mathbf{f}$ TEM images of profuse bundles of SWNTs collected after $\sim 10 \mathrm{~s}$ growth times under these conditions. $\mathbf{g}$ Gated ICCD images of Rayleigh scattering from the vortex rings at the designated times inside a windowed tube furnace obtained using a second, time-delayed laser. $\mathbf{h}$ Several individual SWNTs resulting from time-restricted growth to $<100 \mathrm{~ms}$, each $<100 \mathrm{~nm}$ long, protrude from $<20 \mathrm{~nm}$ NiCo nanoparticles which are embedded in unconvereted carbon. [(c) and (g) reproduced from [37, 38]

metal. Rayleigh scattering images of the propagating vortex rings (e.g., see Fig. 7.4g) revealed that, depending upon the time spent within the hot furnace, SWNTs grow over extended periods of time at rates of $1-5 \mu \mathrm{m} / \mathrm{s}$. Stop-growth experiments for times $\Delta \mathrm{t} \sim 100 \mathrm{~ms}$ revealed only very short $(<250 \mathrm{~nm}$-long) SWNTs as shown in Fig. 7.4h, meaning that the majority of SWNT growth occurs for extended times (hundreds of milliseconds to seconds) [38]. The SWNT products of this condensed phase catalyst-assisted growth are remarkably uniform. Large bundles of SWNTs, shown in Fig. 7.4e, $\mathrm{f}$ are found to contain individual SWNTs with remarkably narrow diameter distribution $(1.2 \pm 0.2 \mathrm{~nm})$, despite a large variety of catalyst nanoparticle diameters $(2-20 \mathrm{~nm})$. This mystery of nucleation and growth persists, and will be contrasted with the broad diameter distributions from low-temperature chemical vapor deposition growth described below in Sect. 7.5. 

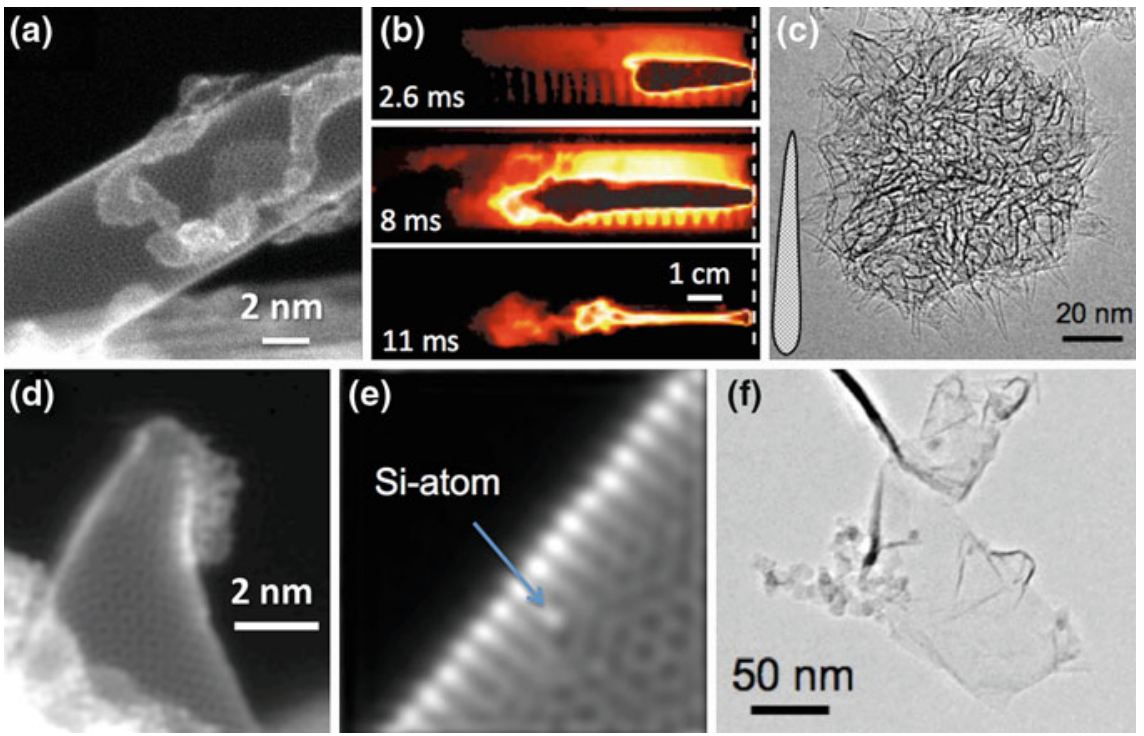

Fig. 7.5 a Z-contrast, Atomic resolution STEM images of single-wall carbon nanohorn with attached graphene flakes. b Side-on images of plasma plumes used for SWNH growth by $10 \mathrm{~ms}-$ long, $100 \mathrm{~J}$ pulses from a Nd:YAG $(1,064 \mathrm{~nm})$ laser on a C target in $1 \mathrm{~atm}$. flowing Ar. c Roughly spherical SWNH aggregate, composed of hundreds of closed nanohorns (subunit depicted as inset). d AR-Z-STEM image of a SWNH tip with attached flake. e AR-Z-STEM image of a SWNH edge, showing lattice incorporation of a single Si atom. f HRTEM image of graphene flake synthesized under similar conditions, although with hydrogen in the background gas

\subsubsection{Single-Wall Carbon Nanohorns}

Like giant fullerenes, single wall carbon nanohorns (SWNHs) are hollow, singlewalled carbon nanostructures that are grown in the highly nonequilibrium conditions of laser plasmas or electric arcs where pure carbon is vaporized into background gases. Discovered in 1999, [7] SWNHs are extended tubular structures with conical, horn-shaped tips as shown in Fig. 7.5. As schematically diagrammed in Fig. 7.5c, the individual SWNH units pack into agglomerates which are roughly spherical in shape, with many protruding ends. They are formed efficiently in high yields by high power laser vaporization into room-temperature background raregas ambients. Diagnostics of the synthesis process, as shown in Fig. 7.5b, have been performed to characterize the growth environment [39-41], and it has been shown that $\mathrm{SWNHs}$ grow at $\sim 1 \mathrm{~nm} / \mathrm{ms}$ growth rates (equivalent to the $1 \mu \mathrm{m} / \mathrm{s}$ growth rate of SWNTs with catalyst-assistance) [42].

Recently, atomic resolution Z-contrast scanning transmission electron microscope (AR-Z-STEM) images have revealed the atomic structure of carbon nanotubes, nanohorns, and graphene sheets-making it possible to directly observe 
defects, both structural and substitutional. For example, in Fig. 7.5a, d, and e it is possible to see the 5-7 Stone-Wales defects responsible for the curvature of the SWNHs. Because Z-contrast STEM utilizes an annular detector, the intensity of the image is proportional to atomic density. For single atoms, such as the single $\mathrm{Si}$ atom embedded in the SWNH in Fig. 7.5e, heavier atoms appear brighter. Singleatom electron energy loss spectroscopy (EELS) can be used to identify the atoms and the nature of their bonding using characteristic near-edge structure [43].

However, equally interesting is the atomic structure of the carbon that did not convert to nanotubes or nanohorns. As shown in Fig. 7.5a and e, carbon adhering to the walls and tips of the nanotubes and nanohorns are not 'amorphous' carbon, but in most cases are apparently well-formed or defective small platelets of singlelayer graphene. These products are either the results of a competing reaction pathway, or intermediates in the synthesis pathway for nanotubes and nanohorns. It has long been speculated that such small and possibly defective graphene flakes might be the feedstock for the growth of larger structures. In support of this argument, annealing experiments of the carbon soot collected after electric arc and laser vaporization of solid carbon (or carbon/metal) have shown the ability of closed, nanohorn-like structures to grow from pure carbon soot, and much longer SWNTs to grow from short 'seeds' of SWNTs that have already nucleated from metal catalyst nanoparticles that are embedded in carbon soot, such as those shown in Fig. 7.4h [44-46]. Therefore, exploration of the remarkable and facile synthesis of closed single-wall carbon structures such as SWNHs in high-temperature laser plasmas is still in its infancy. Can high quality graphene sheets grow in such nonequilibrium conditions? Preliminary experiments have shown that graphene platelets can been synthesized, as shown in Fig. $7.5 \mathrm{f}$ through the addition of small quantities of hydrogen in an attempt to stabilize the dangling bonds thought responsible for the sealing of the graphene platelets during synthesis. Such small sheets can be used as 'building blocks' to construct new nanostructures such as metal-encapsulated "nanooysters" - metal quantum dots within hollow metal shells_-by mixing reactive metal nanoparticles and laser annealing [47]. Highsurface area novel nanocarbons such as nanotubes, graphene, and nanohorns are highly promising supports for metal nanoparticles in catalysis or hydrogen storage [48], or electrodes in batteries and supercapacitors [49].

\subsection{Laser Diagnostics of Single-Wall Carbon Nanotube Growth by Chemical Vapor Deposition}

Although laser vaporization is a highly versatile, nonequilibrium method for the discovery of nanomaterials, chemical vapor deposition (CVD) is the method of choice for nanomaterial growth wherever possible in order to take advantage of well-established wafer scale processing fabrication technology. CVD has the potential to deterministically grow well-aligned nanomaterials in precise locations with controlled lengths and properties. As described above for laser vaporization 
of SWNTs, metal 'catalyst' nanoparticles with an affinity for carbon can grow carbon nanotubes with diameters nearly matching their own. In CVD, the metal nanoparticles typically perform a dual role, first to crack the hydrocarbon gas, and second to assist in the assembly of a carbon nanotube. Two scenarios are considered: (1) vapor-liquid-solid growth (also known as the dissolution/precipitation model) wherein carbon dissolves in the metal nanoparticle (either liquid or solid) and precipitates due to a gradient in the chemical potential, or (2) surface growth, wherein carbon is assembled essentially on the surface of the metal nanoparticle to form sheets, the nanoparticle essentially acting as a form that guides a tubular sock.

Despite the huge body of work on the chemical vapor deposition of carbon nanotubes, many questions remain about nanotube nucleation and growth. The questions generally concern either the factors that control the overall efficiency of the growth process with implications for large scale production, or the factors that govern the exact structure of an individual nanotube with implications for electronic devices. SWNTs have been the focus of effort due to their applicability of semiconducting SWNTs as single electron transistors, and metallic nanotubes as interconnects or transparent conductors. The factors that control the precise crystal structure of SWNTs (their chirality, which determines their electronic structure) at nucleation are not known, however it is clear that once nucleated SWNTs continue to grow with the same chirality up to centimeters in length [50]. The reason that chirality changes do not occur more frequently is that the energy cost to introduce numerous defects simultaneously is prohibitively large. Therefore nanotube nucleation, the initial formation and liftoff of a nanotube "cap", is a crucial process which remains poorly understood, although environmental TEM studies have given tantalizing views of the process [51]. Is it an epitaxial process wherein the carbon atoms precisely register with a crystalline (solid) metal nanoparticle, or are the nanoparticles liquid during nucleation and subject to random thermal fluctuations (thereby implying that the controllable synthesis of nanotubes with prescribed chirality is impossible). Evidence seems to indicate that both solid and liquid nanoparticles can grow carbon nanofibers and nanotubes, so hope is still alive that chirality control is possible through proper control over the synthesis of metallic nanoparticles. In addition, selectivity has been achieved in the preferential growth of metallic vs. semiconducting SWNTs on miscut substrates (beyond their $1 / 3: 2 / 3$ ratio expected based upon random fluctuations).

Resonance Raman spectroscopy has quickly become a well-developed method to remotely characterize the diameter and chirality of single SWNTs [52, 53]. In SWNTs, the density of electronic states is bunched close to the van Hove singularities of these one dimensional structures. When the incident laser wavelength is tuned in resonance with van Hove singularities in the valence and conduction bands of a SWNT, the Raman scattering cross section becomes very large as electrons and phonons couple strongly under these conditions. Single SWNTs are observable, including their characteristic radial breathing mode (RBM), G-band (graphite-related optical mode), D-band (defect-induced), and G' band (overtone of the D-band). Metallic and semiconducting SWNTs are easily distinguished by 

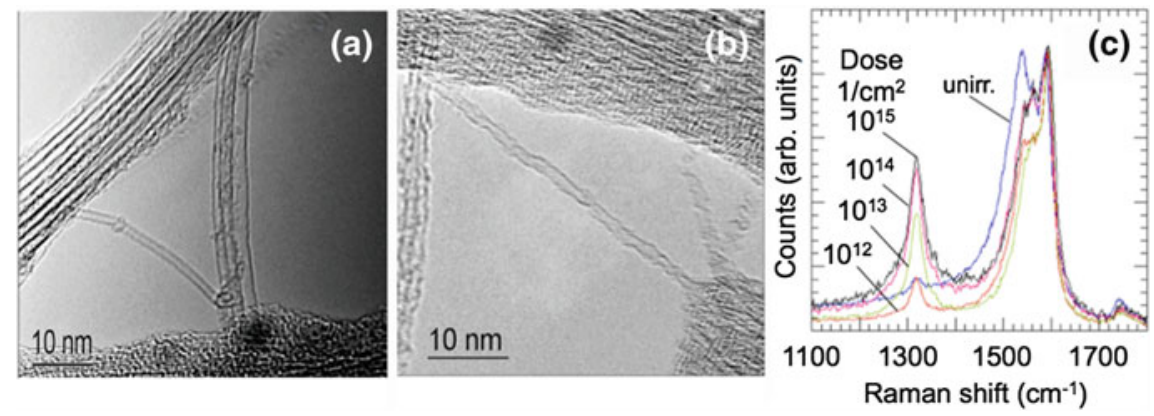

Fig. 7.6 TEM images of SWNTs produced by laser vaporization $\mathbf{a}$ before and $\mathbf{b}$ after ion-beam irradiation by $\mathrm{Ar}^{+}(5 \mathrm{keV})$ with well-defined doses between $10^{12}$ and $10^{15} \mathrm{~cm}^{-2}$, correlating to an estimated $0.01-10$ defects per nanometer of nanotube length. c Raman spectroscopy $\left(\lambda_{\text {ex }}=\right.$ $633 \mathrm{~nm})$ of corresponding samples show the evolution of the D-band $\left(\sim 1,320 \mathrm{~cm}^{-1}\right)$ with induced defect density. The G-band shows more pronounced quenching of the metallic SWNTs $\left(\sim 1,540 \mathrm{~cm}^{-1}\right)$ than the semiconducting SWNTs $\left(\sim 1,590 \mathrm{~cm}^{-1}\right)$ with evolving dose. Raman spectroscopy was also used to observe healing of the damage by heat treatments (not shown). High doses $\left(>5 \times 10^{13} \mathrm{~cm}^{-2}\right)$ lead to irreversible damage as evidenced by HRTEM observation, a broadening of the D-band width approaching that of amorphous carbon, and the inability to reduce the D-band intensity to that of pre-irradiation values

the shape of their G-band (graphite-related optical mode) in the 1,500-1,605 $\mathrm{cm}^{-1}$ range. The diameter of the nanotube (in $\mathrm{nm}$ ) is $\sim 248 /\left(\mathrm{RBM}_{\mathrm{in}} \mathrm{cm}^{-1}\right)$. In fact, the exact chiral indices $(n, m)$ of the nanotube may be determined by careful comparison of the resonance Raman condition with theoretical predictions [52, 53]. Very recently, the growth rates of individual SWNTs with different chiral indices were measured in situ during chemical vapor deposition using resonance Raman scattering performed at high temperatures [54]. The technique inferred the growth rate of the nanotubes from the increase in the G-band intensity. The measured growth rates agreed with recent theoretical predictions of a chirality-dependent growth rate [55]. Raman spectroscopy is more commonly used with a variety of fixed-wavelength lasers to characterize the overall diameter distribution and level of defects in ensembles of SWNTs.

For example, Fig. 7.6 shows TEM images and Raman spectra of SWNTs (a) before and (b) after the introduction of defects with well-defined doses of $\mathrm{Ar}^{+}$ $(5 \mathrm{keV})$ ions. With 633-nm laser excitation, resonances exist with both metallic and semiconducting SWNTs within the 1.2-1.4 nm diameter distribution in the sample, as evidenced by the two-peaks in the G-band. As defects are introduced with doses of $10^{12}-10^{15} \mathrm{Ar}^{+}$ions $\mathrm{cm}^{-2}$ the $\mathrm{D}$-band is seen to rise and saturate, while the G-band of the metallic SWNTs decreases much more strongly than that of the semiconducting SWNTs. Using this technique to monitor the level of defects in damaged SWNTs it was observed that annealing could reduce the D-band intensity to pre-irradiation values only for $\mathrm{Ar}^{+}$doses $<5 \times 10^{13} \mathrm{~cm}^{-2}$, above which irreversible damage was incurred. 
(a)

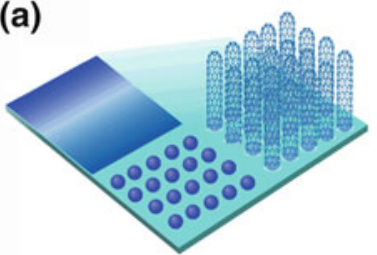

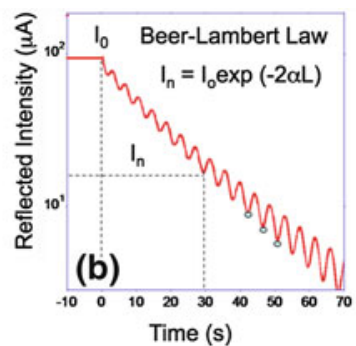

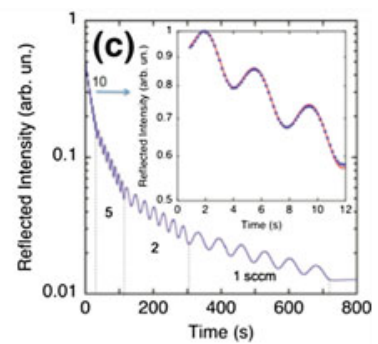

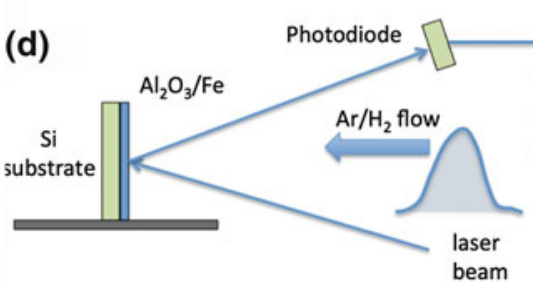
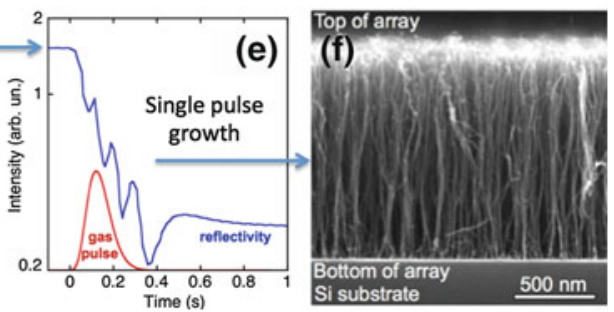

Fig. 7.7 a Schematic of vertically-aligned nanotube array (VANTA) growth by CVD. Thin metal films roughen into nanoparticles attached to the substrate, from which aligned arrays of nanotubes grow upon exposure to hydrocarbon gas at high temperature. b The reflected intensity of a laser from the growing nanotube array decreases exponentially with length, L, due to the effective extinction coefficient, $\alpha$. Fringes result from optical interference from light reflected by the substrate and the top surface of the array. $\mathbf{c}$ In situ monitoring of VANTA growth starting at $10,5,2$, and $1 \mathrm{sccm}$ acetylene flow. The growth rate can be estimated at $300 \mathrm{~nm} /$ fringe. d Experimental setup for pulsed CVD of VANTAs. e Time dependence of a single 0.2-s CVD gas pulse amplitude ( $r e d$ ) arriving at the substrate and the corresponding change in laser reflectivity (blue) resulting in a 1.3- $\mu$ m-tall VANTA in (f) which shows a side-view cross section of the array grown in $\sim 0.6 \mathrm{~s}[(\mathbf{c})$ and (f) from 65$]$

One of the most interesting and widely studied ensembles of nanotubes are vertically aligned nanotube arrays (VANTAs, also known as carpets or forests). As schematically shown in Fig. 7.7a, these forests are typically grown by CVD from thin films of metal which dewet into densely-packed nanoparticles. With sufficiently high nucleation density, nanotube forests self-assemble as the individual nanotubes grow into dense, self-supporting, oriented arrays that can be grown to centimeter lengths. The VANTA platform of vertically-oriented, continuous nanotubes is highly promising for a wide range of different applications such as gas sensors [56], flexible electronics and field emission devices, vertical interconnects for microelectronics [57], thermal interface materials [58], heat pipes [59], and unique optical absorbers. The unique morphology of VANTAs has been shown to be especially well suited for "gecko" type adhesives, super-compressible foams, and carpets from which fibers and transparent, conductive sheets [60] can be continuously spun. VANTAs can be synthesized at "super growth" rates [61] such that a millimeter tall forest can grow within ten minutes, thereby enabling mass production of this unique, aligned nanotube architecture. The forests tend to grow in a coordinated growth mode despite the disparity of nanotube diameters 
and individual growth rates present in the VANTA arising from catalyst nanoparticles of different size and catalytic activity. Typical arrays are only $\sim 5-10-$ vol. $\%$ dense, corresponding to areal nanotube densities of $5 \times 10^{11} \mathrm{~cm}^{-2}$. However, through reduction of catalyst particle size and spacing, small-diameter SWNTs packed in VANTAs with areal nanotube densities up to $10^{13} \mathrm{~cm}^{-2}$ have been reported [62].

Laser-based interferometry and absorption have proven to be invaluable realtime diagnostics of VANTA growth kinetics under actual synthesis conditions [11, 63-67]. As shown in Fig. 7.7d, laser light is reflected from the substrate on which a buffer layer (typically $\mathrm{Al}_{2} \mathrm{O}_{3}$ ) and metal catalyst film is deposited. As the nanotubes nucleate and grow upon exposure to the CVD gases at high temperatures, optical absorption and interference occurs as indicated in Fig. 7.7c. Interference oscillations occur due to constructive and destructive interference between light reflected from the top of the growing forest, and light which has penetrated the forest and reflected from the substrate. Depending upon the index of refraction of the array and the laser wavelength employed, each fringe corresponds to a discrete amount of array growth (e.g., $300 \mathrm{~nm} /$ fringe in Fig. 7.7b, c). As indicated in Fig. 7.7c, changes in the growth rate (such as those occurring from changing the feedstock supply) are immediately apparent in the fringe spacing. The absorbance of the array corresponds to an effective extinction coefficient. Since the arrays typically grow from their bottoms where the catalyst nanoparticles are anchored to the substrate, it is important to note that each additional fringe and attenuation of the signal that occurs reflects the incremental growth of a layer at the bottom of the array, providing a step-by-step record of each layer of the array. The diagnostic technique and analysis has been described in detail in [11]. Such measurements form the basis for a complete rate equation-based model of the nanotube CVD process which can predict, from a few measurements at different temperatures during growth test runs with a particular catalyst system, subsequent growth behaviors including: (1) growth rate, (2) number of walls of the nanotubes in the array, (3) the terminal length of the array (4) the growth kinetics versus feedstock flux. The interferometry technique is typically applicable over approximately four orders of magnitude in signal attenuation, which corresponds to roughly the first 20 microns of growth. For growth to millimeter lengths, direct optical imaging is used to measure the kinetics.

Two of the most important parameters governing the properties and applicability of VANTAs are their length and density. The density of VANTAs appears to be integrally linked to their cooperative growth mechanism, which is still not understood despite a number of interesting studies in this area. Due to variations in metal catalyst nanoparticle size and their interactions with the substrate, it is reasonable to expect that the nanoparticles which support nanotube growth have different catalytic activities, with some fraction capable of maintaining growth of a nanotube for a very long time under favorable conditions. Ostwald ripening of metal catalyst nanoparticles $[68,69]$ during the VANTA growth process can result in a change in nanotube density and diameters, and diffusion of the metal 
nanoparticles into the substrate can occur [70], leading to further decrease in array density over extended times.

Recently, laser reflectivity and attenuation coupled with fast pulsed gas delivery have revealed the nucleation and growth kinetics of VANTAs on rapid timescales, and have also revealed that the density and diameter of nanotubes in VANTAs are directly related to the feedstock flux [65-67]. As shown in Fig. 7.7d-f, pulses of gas delivered from a pulsed valve within a tube furnace can nucleate, grow, and terminate growth of aligned nanotube arrays within $\sim 0.5 \mathrm{~s}$. By using pulsed gas delivery at low pressure, delays in nucleation can be observed with sub $0.1 \mathrm{~s}$ resolution, and the kinetics of the nucleation and growth process can be recorded by the interference oscillations and attenuation described above [67].

Using pulsed CVD, it was discovered that the nucleation time for growth decreased over three orders of magnitude with increasing flux (i.e., partial acetylene pressure) in the pulse [66]. Moreover, it was discovered that the changing flux within each pulse induced significant density changes within the arrays [65]. Figure 7.8 summarizes the results. As shown in the SEM micrograph of Fig. 7.8d, repetitively pulsing the acetylene feedstock results in nanotube arrays with a distinct striped appearance, the contrast variation (shown in yellow) resulting from the fluctuation in density within each layer. As shown in the TEM and Z-contrast STEM micrographs of exfoliated strips of the array representing the first three gas pulses in Fig. 7.8a and b, respectively, the density of the arrays varies dramatically within each gas pulse. The Z-STEM image is directly proportional to carbon density, and the profile of the array is shown in blue in Fig. 7.8c. The density of the array is seen to vary by up to a factor of 1.6. The red trace in Fig. 7.8c is the effective extinction coefficient obtained by analyzing data similar to that in Fig. 7.7e for each pulse. By simultaneously estimating the increase in array length versus time from the interference fringes, a correlation between extinction coefficient and length is obtained. Therefore, the effective extinction coefficient is directly proportional to the density in growing VANTAs, providing a key real-time diagnostic [65].

By analyzing the real-time kinetics of the growth process pulse by pulse, the growth rate and efficiency of the nanotube ensemble is seen to drop on each successive growth event, as shown in Fig. 7.8e. This is also reflected in the decreasing length of each stripe from the top to the bottom of the array. Moreover, detailed analysis of the nanotubes within each array (by both Raman spectroscopy and ex situ HRTEM analysis) revealed that high-flux pulses tended to extinguish the small-diameter nanotubes within the ensemble, while low flux pulses permitted the full spectrum of nanotube diameters to renucleate and regrow on successive pulses.

Two distributions of nanotubes were observed in SEM images of the arrays: continuous nanotubes which continued throughout all of the layers, and disordered nanotubes at the top (high flux portion) of each layer. These smaller-diameter nanotube products apparently grew, terminated growth, and were ripped off and carried off by the continuously-growing, larger-diameter nanotubes. This additional 

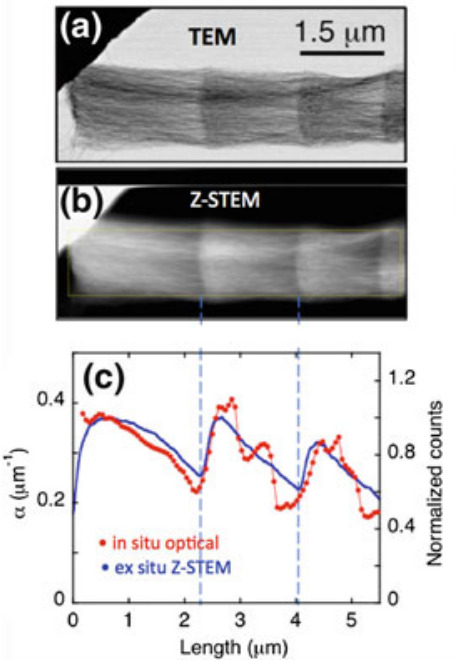
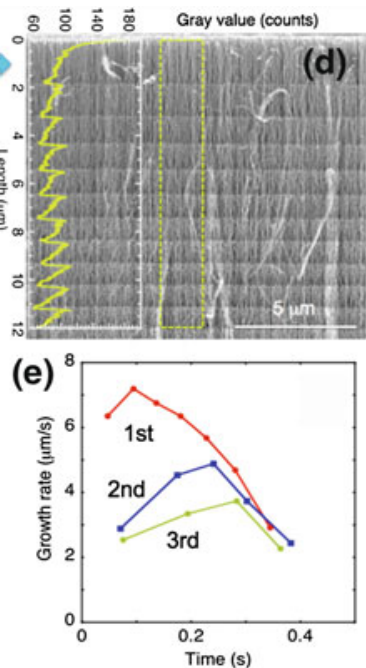

(f)

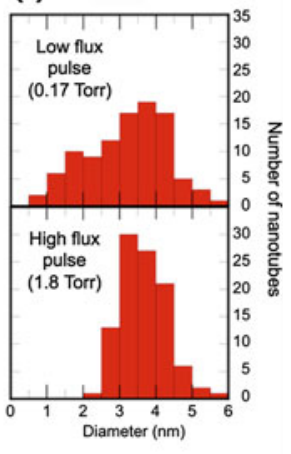

Fig. 7.8 (a) TEM and Z-contrast STEM images of a strand of VANTA grown by successive pulses of gas. The Z-STEM image in (b), when integrated within the yellow box yields the blue curve in (c) which is directly proportional to the density of the nanotubes in the array, which is seen to vary by a factor of 1.6. The red curve in (c) is the density in the array inferred from the extinction coefficient $\alpha(\mathrm{L})$ calculated from the measured $\alpha(\mathrm{t})$ and array length $\mathrm{L}(\mathrm{t})$. (d) Such density variations lead to the striped appearance in SEM images of VANTAs grown by pulsed CVD. The integrated intensity in the dashed zone yields the intensity variations shown at left. (e) Growth rate changes during the first 3 gas pulses measured from laser interference fringe spacings versus time. (f) Nanotube diameter distributions measured by TEM analysis for low- and high-flux gas pulses, showing that high-flux pulses result in the extinction of nanotubes with $<2 \mathrm{~nm}$ diameter [from 65,66 ]

distribution of "crossbar" nanotubes, oriented randomly during the initial growth region of each pulse, produced the observed variation in density.

Through the in situ diagnostics provided by the real-time laser reflectivity, the gas flux could be lowered to produce VANTAs without stripes, density changes, or variations in diameter within the array. Moreover, the dependence of catalyst nanoparticle nucleation efficiency on feedstock flux and the origin of the coordinated growth mode was probed by lowering the flux in each gas pulse. Nanotubes were shown to nucleate and then renucleate repeatedly, to grow "digitally" in incremental fashion on successive gas pulses [67]. Figure 7.9a shows the in situ laser reflectivity curves for two growth runs where clear interference oscillations confirm that the nanotube arrays are growing in coordinated fashion. Each gas pulse causes a segment of the overall growth curve, as shown in the inset to Fig. 7.9a, corresponding to an incremental growth of $\sim 20 \mathrm{~nm} /$ pulse. However, at least $\sim 60-100 \mathrm{~nm}$ of nanotube growth is required before nanotubes self-align into a vertical array. How can one tell if the nanotube nucleation efficiency is sufficient to produce an aligned array? 
(a)
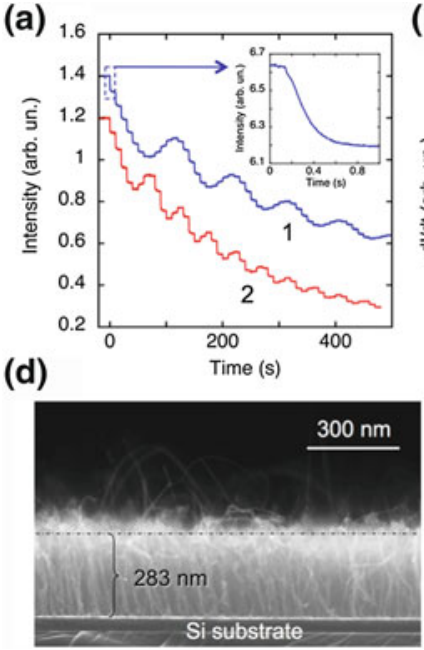

(b)

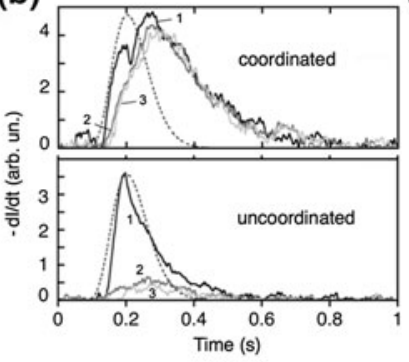

(e) (c)

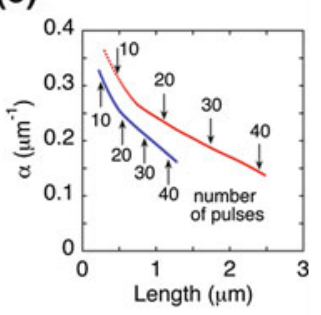

Fig. 7.9 In situ laser reflectivity diagnostics of the incremental, pulsed CVD growth of aligned nanotube arrays. a Reflected laser intensity during two different growth runs using multiple gas pulses to multiply stop and start the growth of SWNTs (inset shows a single step, corresponding to growth on the first gas pulse). Fringes indicate aligned array growth, $\sim 20 \mathrm{~nm} / \mathrm{pulse}$. b Derivatives of laser reflectivity signal for pulses 1,2,3 as compared to gas pulse (dashed line) in cases where growth is (top) coordinated and aligned and (bottom) uncoordinated. c Changes in the density of the nanotubes grown on each pulse as measured by optical extinction coefficient, $\alpha$, as a function of pulse number and cumulative length of the array. d Side-view SEM of a 283-nm aligned array grown incrementally by pulsed CVD. e TEM image of SWNT, showing no visible evidence of being grown incrementally in 20-nm steps. f Diameter distribution of SWNTs in the array measured by TEM [from 67]

Inspection of the derivative of the reflectivity curves on each pulse, $d I / d t$, yields the instantaneous growth rate. Two behaviors were observed corresponding to coordinated and uncoordinated growth, as illustrated in Fig. 7.9a and b. For arrays which would later exhibit interference oscillations indicative of coordinated, aligned growth, the first exposure to the acetylene pulse produced a double peaka fast ( $\sim 50 \mathrm{~ms}$ ) peak followed by a broader, delayed peak, which extends beyond the tail of the modeled gas pulse. All subsequent pulses show only the second peak. The relative intensities of these two peaks vary considerably, depending on the growth run. In the case of uncoordinated growth, the intensity of the second peak is relatively low and appears as a shoulder on the pronounced first peak of the first gas pulse. Moreover, the total width is comparable to the calculated gas pulse width and is narrower than that for coordinated growth. In these cases, growth typically stops quickly as indicated by the lack of reflectivity changes after a few additional gas pulses.

Practically, these in situ laser reflectivity diagnostics afford the opportunity to grow ultrashort SWNT arrays digitally to prescribed lengths $>60 \mathrm{~nm}$ with $\pm 20 \mathrm{~nm}$ accuracy, as shown for the 283-nm VANTA in Fig. 7.9d [67]. 


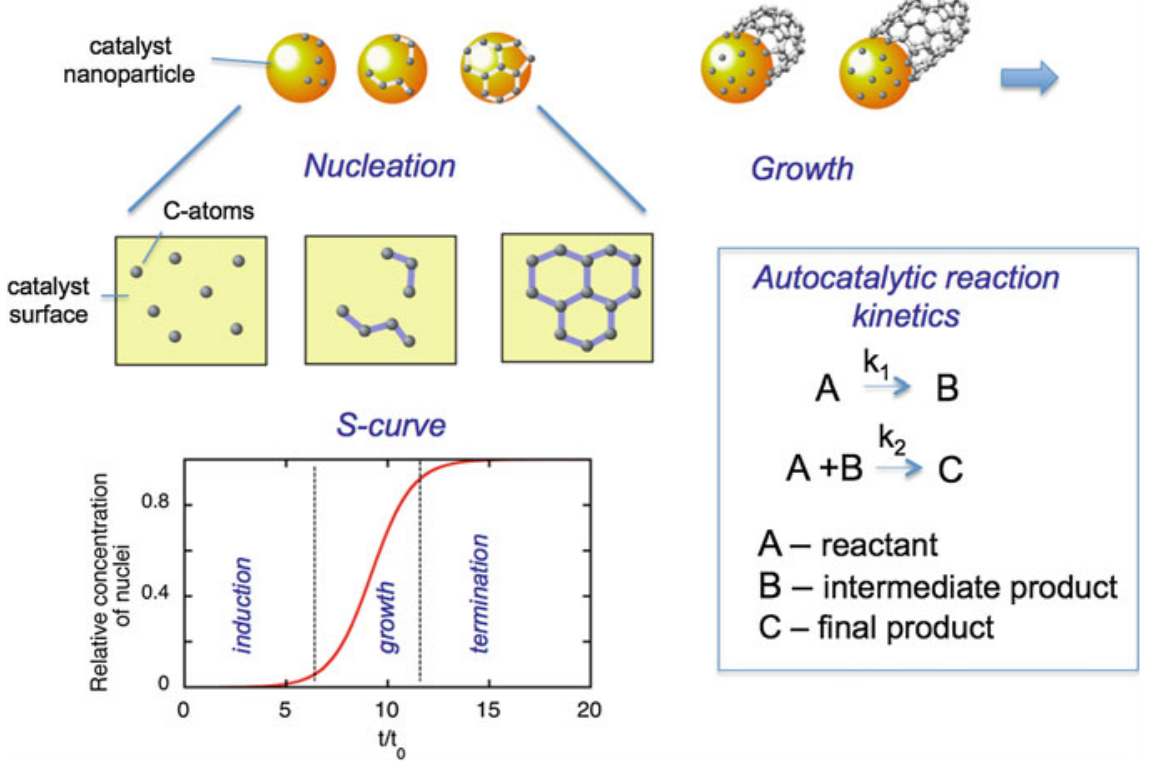

Fig. 7.10 Diagram indicating the kinetic evolution of carbon nanostructures based upon fluxdependent, time-resolved laser diagnostic measurements of the CVD process. Measured kinetics indicate that $S$-shaped growth curves indicative of autocatalytic chemical reactions representing induction, growth, and termination periods. Intermediate products react with reactants to accelerate the formation of closed (graphenic) carbon structures on a catalyst nanoparticle surface. For nanotubes, cap liftoff and continued growth can continue this process over multiple pulses

Importantly, the SWNTs show no evidence of stopping and restarting growth (Fig. 7.8e) with no visible diameter changes or defects on these length scales. Moreover, the entire diameter distribution is preserved (Fig. 7.9f). Such ultrashort SWNT arrays may be useful for thermal interface materials, interconnects, field emitters, sensors, and other applications. It should be noted, however, that as in the pulsed growth of long VANTAs the nanotube density drops with repeated renucleation events, as indicated by the drop in the optical extinction coefficient per pulse in Fig. 7.9c. By comparing the drop in density per pulse it can be estimated that over $98 \%$ of all nanotubes regrow on each successive pulse [67].

The observed double-peak feature in the evolution of the growth rate, $d I / d t$, can be interpreted in the context of autocatalytic kinetics for both nanotube nucleation and growth where in both cases induction delays are explained by the time required to form intermediate species which accelerate chemical conversion of the feedstock gas. Such autocatalytic kinetics result in "S-shaped" growth curves as shown in Fig. 7.10, with a slow induction period during the buildup of the necessary intermediates, a period of rapid and efficient reaction, and finally a period of decline as the chemical reactions terminate due to lack of reactants or available sites. Such "S-shaped" growth kinetics have been observed in carbon fiber [71] 


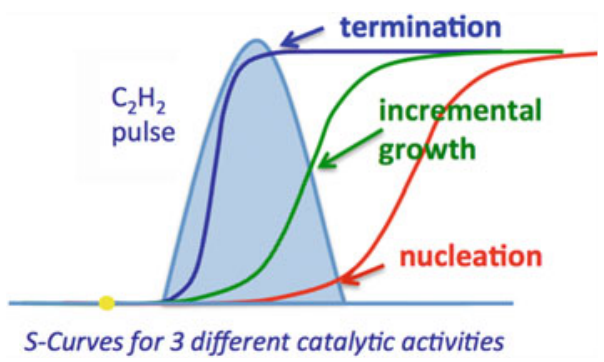

Fig. 7.11 Schematic summarizing the results of the laser reflectivity and pulsed CVD nanotube growth experiments in the context of autocatalytic kinetics model, where growth proceeds in an $S$-shaped curve, with a nucleation period, a growth period, and a termination period due to the requirement for the formation of intermediate reactants. Nanoparticles with different activity catalyze the growth of nanotubes at different rates, leading to either barely nucleation $(\mathrm{red})$, rapid growth and termination (blue), or repeated nucleation and growth (green) during each gas pulse

and nanotube [72] growth experiments for years, and more recently in the growth of carbon nanotube arrays [73-75]. However, separating the kinetics of nucleation and growth has been difficult until now.

Recently, Latorre et al. [76] developed a phenomenological autocatalytic model to describe "S-shaped" kinetics in the growth of nanotube forests measured by in situ Raman spectroscopy. The model used the framework of growth by dissolution-precipitation driven by the gradient of carbon concentrations at the metal catalyst nanoparticle surface. First, however, the chemical reactions required for the nucleation and growth of a stable nanotube "cap" were considered, followed by the processes for cap liftoff and nanotube growth. The termination phase for nanotube nucleation resulted from the lack of available sites on a catalyst nanoparticle, while the collection termination of nanotube growth in the arrays could be due to a variety of different reasons including Ostwald ripening of the catalyst, [69] mechanical stress, [77-80] catalyst particle overcoating [11, 81], but also including chemical reactions, such as those discussed by Eres et al. to explain the preferential growth of nanotube arrays with acetylene as the primary precursor [74] and proposed mechanisms for the self-assembly of nanotubes via acetylene by autocatalytic reactions [75].

This latter model involving autocatalytic chemical reactions explains the pulsed CVD nanotube growth kinetics measured by laser reflectivity in the context of Fig. 7.11. Ensembles of catalyst nanoparticles are likely to contain a range of activities for the decomposition of feedstock gas and the processing of carbon into nanotubes. Our studies of the growth kinetics reveal S-shaped curves which are characteristic of autocatalytic chemical reactions which imply intermediate states that are required to accelerate the rapid growth region after the nucleation period. The S-curves represent a nucleation period, a period of rapid growth, and a termination period where particles may get overcoated with carbon, for example. Higher fluxes tend to activate chemical reactions on each nanoparticle, the most active of which may undergo their entire life cycle within the gas pulse lifetime. 
Therefore, at the highest fluxes, a variation in density is explained to yield the arrays in Fig. 7.8, wherein smaller catalyst nanoparticles nucleate, grow, and terminate small nanotubes in the early part of the acetylene gas pulse (blue curve in Fig. 7.11) while larger nanoparticles and their corresponding nanotubes survive the high flux conditions to incrementally grow pulse after pulse. Similarly, at lower fluxes, termination of growth for the particles of both high and low catalytic activity can be avoided to grow continuous nanotubes incrementally over a wide range of nanotube diameters, as shown in Fig. 7.8 [66, 67].

\subsection{Graphene and Beyond: Laser Processing for 2D Layered Materials}

Two dimensional materials just one to several atomic layers thick, but extending to macroscopic dimensions, are frontier mesoscale materials that promise to enable a wide range of practical applications. Stimulated by the rapid progress in the experimental synthesis and exploration of single layer graphene (SLG) and few layer graphene (FLG), similar efforts are now being applied to obtain ultrathin specimens of other familiar 2D materials such as $\mathrm{MoS}_{2}$ and other chalcogenides [82-85], h-BN [86], transition metal oxides and hydroxides [87], and compounds such as $\mathrm{Bi}_{2} \mathrm{Te}_{3}$, and $\mathrm{Bi}_{2} \mathrm{Se}_{3},[3,88]$ and $\mathrm{GaSe}$ [89]. Here, we will briefly outline the areas of future research where laser interactions with these materials should play key roles in their synthesis, processing, and remote characterization.

The rapid exploration of the unique properties of graphene were stimulated by the transfer of small flakes of that were mechanically exfoliated from highlyoriented pyrolytic graphite (HOPG) [90]. The outstanding electrical conductivity (mobilities up to $15,000 \mathrm{~cm}^{2} \mathrm{~V}^{-1} \mathrm{~s}^{-1}$ ), zero band gap, and near optical transparency $(98 \%)$ of the ultrathin material appear promising as a flexible, transparent, conductive electrodes for a variety of applications including touch panels, displays, and flexible organic electronics such as organic photovoltaics, light emitting diodes, and transistors.

\subsubsection{Mechanical and Chemical Exfoliation Methods and Laser Processing}

To realize such large-scale applications, methods must be developed to produce large areas of high-mobility nanosheet architectures. Conductivity across junctions between individual nanosheets is a key problem, requiring large individual grains. However, large-scale production of exfoliated material typically involves techniques such as sonication in liquids. 2D nanosheets produced in this way are typically small ( $<150 \mathrm{~nm}$ in diameter) and can suffer damage to their structure and 
properties [91]. Finding controllable methods to exfoliate layered materials without damage is a major challenge where laser-surface interactions may play a key role. Laser exfoliation of HOPG in liquids for the production of microns-sized flakes of FLG has been demonstrated using $532 \mathrm{~nm}$ irradiation at $1.0 \mathrm{~J} \mathrm{~cm}^{-2}$ which was thought to occur through the compression and expansion of the HOPG surface [92]. Similarly, 248-nm laser exfoliation of $\mathrm{WS}_{2}$ in water was demonstrated to result in microns-sized multilayer flakes, presumably due to laser induced shock waves and the possible intercalation of $\mathrm{OH}$ and $\mathrm{H}$ radicals created by the two-photon dissociation of water [93]. Recent theoretical predictions indicate that ultrafast excitation of graphite might induce exfoliation of intact graphene layers by inducing spillout of electrons and subsequent Coulomb repulsion between the top layers [94]. The controllable deposition of laser energy may therefore hold unique processing advantages.

Chemical treatments are alternate or auxiliary methods for exfoliation. For example, graphite oxide (GO) is hydrophilic and can be easily exfoliated in water. GO is produced (by oxidizing graphite with sulfuric acid, sodium nitrate, and potassium permanganate) in the Hummers method [95]. The processed material has a disordered, buckled structure with various types of oxygen-containing functional groups (e.g., epoxide, carbonyl, hydroxyl, phenol). The disordered structure of GO varies due to carbon:oxygen ratios variation between 2.1 and 2.9 [95]. Alternatively, GO can be thermally exfoliated by rapidly evolving the oxygen-containing groups such that pressure exceeds the van der Waals forces holding the layers together [96]. In both cases, after exfoliation of GO, functionalized single-layer graphene oxide can be obtained which must be reduced, either chemically [97] or with prolonged thermal treatments, to provide graphene-like layers for applications. Raman spectroscopy and scanning transmission electron microscopy [98] shows that this material is still highly defective, however.

Recently, laser-based reduction of dry GO has been demonstrated using CW and pulsed 532-nm laser irradiation [99] or 248-nm irradiation to directly write graphene lines and patterns [100]. The use of lasers to tunably desorb functional groups alter 2D material stoichiometry will take advantage of pulsed laser vaporization and desorption studies primarily targeted for pulsed laser deposition of thin films.

In addition, it is often desirable to adjust the number of layers in $2 \mathrm{D}$ nanosheets. Recently, the controllable laser thinning of $\mathrm{WS}_{2}$ nanosheets was demonstrated and verified with Raman spectroscopy [101].

\subsubsection{Laser Interactions in the Synthesis and Characterization of Graphene and other $2 D$ Nanosheets}

The highest quality graphene is synthesized by either top-down or bottom-up processes. Extremely high quality graphene can be obtained by thermally sublimating silicon from silicon carbide substrates in vacuum at temperatures between 
1,000 and $1,500{ }^{\circ} \mathrm{C}$. "Mulilayered epitaxial graphene (MEG)" produced in this way can be tailored to produce ribbons along predetermined step edges or grains along the $\mathrm{Si}$ or $\mathrm{C}$ terminated faces of $\mathrm{SiC}$ with mobilities up to $5,000 \mathrm{~cm}^{2} / \mathrm{Vs}$. The material is epitaxially bonded in places to the $\mathrm{SiC}$ substrate, forming a unique material which is envisioned to be used for a new type of graphene electronics directly on $\mathrm{SiC}$ wafers. Since graphene is a semimetal with no bandgap, new types of ambipolar transistor are being developed [102].

Laser heating and decomposition of $\mathrm{SiC}$ to form monolayer, bilayer, and trilayer graphene was demonstrated with different fluences using 500 shots of $\mathrm{KrF}$ laser irradiation in vacuum [103]. More recently, continuous-wave $\mathrm{CO}_{2}$ laser irradiation of $\mathrm{SiC}$ (0001) was demonstrated to induce epitaxial graphene growth on timescales of $\sim 1 \mathrm{~s}$ in ambient Ar atmospheres, enabling the writing of graphene patterns without pretreatments [104]. Laser processing therefore has the advantage of rapid heating and cooling rates, controllable and localized energy deposition, and high instantaneous surface temperatures through the choice of pulse width.

By far, the most widely studied technique for the formation of graphene over large areas is CVD, where conditions nearly identical to those used for the growth of carbon nanotubes are employed. Two types of metal foil or film substrates are used: those with low carbon solubility (e.g., $\mathrm{Cu}$ ) or high carbon solubility (e.g., Ni). On Cu, continuous films of SLG or FLG are formed at $1,000-1,050{ }^{\circ} \mathrm{C}$ from methane decomposition at low or high pressure in flowing hydrogen or hydrogen/argon mixtures, very near the evaporation temperature of $\mathrm{Cu}$ [105]. Graphene islands nucleate and grow in lobed, hexagonal, or square grains to cover the entire surface in a self-limiting fashion [106]. This graphene can be transferred to arbitrary substrates by attaching a polymer (PMMA) to the graphene surface, etching away the $\mathrm{Cu}$ in $\mathrm{FeCl}_{3}$ solution, pressing the graphene to the substrate of choice, and etching away the polymer [107]. This procedure has been demonstrated in roll-to-roll (30") processing onto PET substrates, achieving $125 \Omega /$ square sheet conductivity [108]. $\mathrm{On} \mathrm{Cu}$, large graphene grains can grow across $\mathrm{Cu}$ grain boundaries within the foil while on $\mathrm{Ni}$, the grain orientation of the $\mathrm{Ni}$ drastically affects the nucleation and growth of the graphene.

However, there is significant disagreement in the literature regarding the growth mechanisms of graphene on metals. Especially for metals with high carbon solubility, it is unclear what fraction of growth occurs isothermally at high temperatures [109] and how much occurs during cool down [110, 111]. Like nanotube growth, the debate centers on growth by surface processes versus that by dissolution/precipitation. Several in situ diagnostics are beginning to provide some insight, though [110]. For example, in situ X-ray photoelectron spectroscopy (XPS) [112], and in situ X-ray diffraction (XRD) [113] have shown recently that graphene can grow isothermally on Ni films, and is not limited to the precipitation mechanism during cool down [110].

To answer these questions, real-time optical diagnostics can be performed in situ during growth $[114,115]$. Figure 7.12 a shows a setup for confocal laser microRaman scattering spectroscopy, imaging, and reflectivity utilizing a specially-modified growth chamber for CVD of graphene using a pulsed valve to 

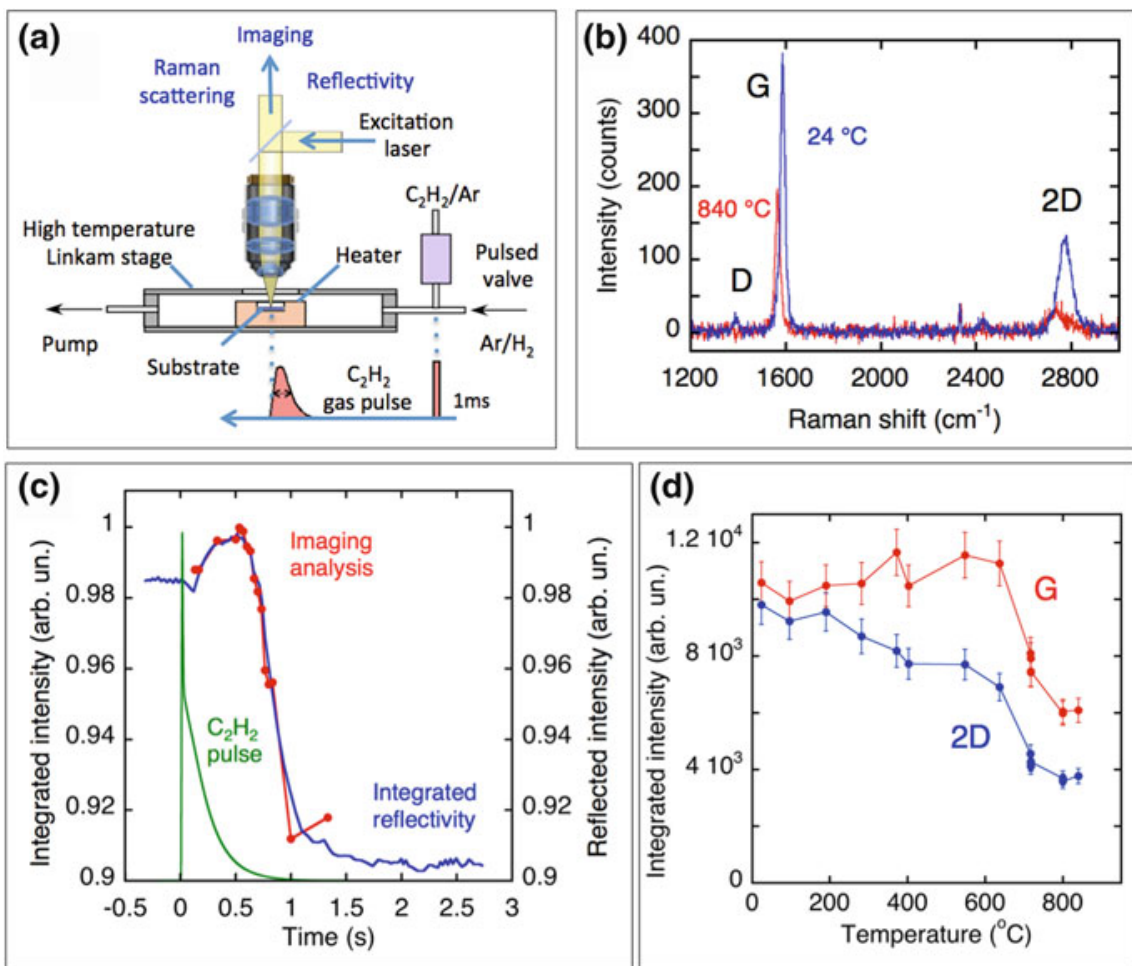

Fig. 7.12 a Setup for confocal laser micro Raman spectroscopy, imaging, and reflectivity studies of graphene growth by pulsed CVD. b Characteristic Raman spectrum of graphene on Ni $\left(\lambda_{\mathrm{ex}}=\right.$ $532 \mathrm{~nm}$ ) showing $D, G$, and $2 D$ bands at 840 and $24^{\circ} \mathrm{C}$. The width and relative $2 D$ : $G$ band ratio can be used to infer the number of graphene layers. c Time-resolved kinetics of graphene growth at high temperature $\left(690{ }^{\circ} \mathrm{C}, \mathrm{C}_{2} \mathrm{H}_{2}\right.$ pulse, $\mathrm{Ni}$ film substrate) from imaging analysis and integrated reflectivity with a photodiode, in comparison with the $\sim 0.2$-s gas-pulse flux (in green). $\mathbf{d}$ Raman $G$ and $2 D$ band integral intensities reveal additional graphene precipitation during cooldown from the growth temperature of $840{ }^{\circ} \mathrm{C}$ [115]

introduce pulses of acetylene growth gas within $0.1 \mathrm{~s}$ [115]. Raman scattered light is dispersed with a spectrometer to look for the appearance of the characteristic Raman spectrum of graphene, shown in Fig. 7.12b. The Raman shift of the 2D band and the ratio of $2 \mathrm{D}: \mathrm{G}$ band intensities can be used to infer the number of graphene layers [116]. As shown in Fig. 7.12c after an induction time following the introduction of the hydrocarbon gas at $840{ }^{\circ} \mathrm{C}$, the reflectivity of the sample increases slightly and suddenly drops due to carbon deposition. Imaging through the microscope shows how the patches of graphene nucleate and grow rapidly at this high temperature, with the integrated intensity matching that from the photodiode. Raman spectroscopy with 1-s acquisition time confirms this sub-second growth of graphene, clearly showing that graphene grows isothermally at high temperatures. By monitoring the integrated intensity of the $\mathrm{G}$ and $2 \mathrm{D}$ bands as the 

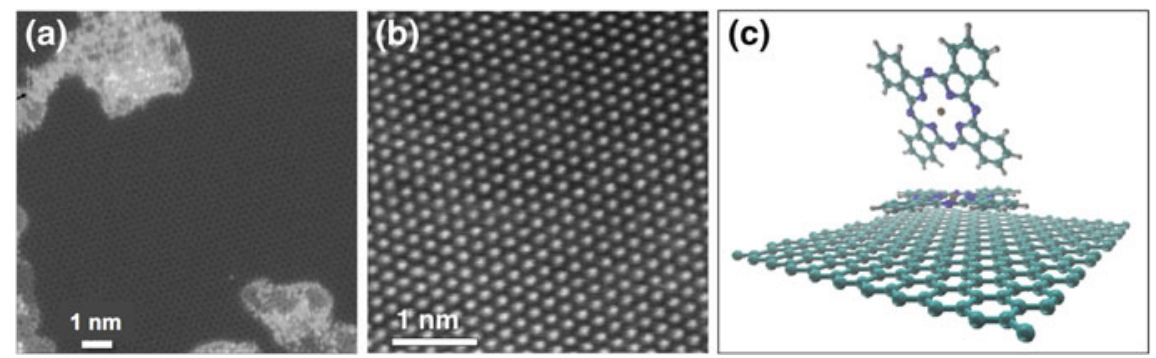

Fig. 7.13 a Aberration corrected Z-STEM image of single-layer graphene (dark area) with residues from the synthesis, etching, and transfer process (bright areas). b AR-Z-STEM image $\mathrm{Ga}$ and Se atoms in an exfoliated single-layer GaSe nanosheet. c Atomistic simulation of copper pthalocyanine molecules interacting with single-layer graphene [(c) from 119]

sample is cooled down, any additional fractional precipitation of graphene from carbon dissolved within the Ni film is revealed by the increase in G-band and 2Dband intensity (Fig. 7.12d). Real-time diagnostics such as these are essential to understand the growth process, and to later serve as real-time diagnostics of graphene nanomanufacturing.

As shown in Fig. 7.12, the growth of graphene can be very rapid on substrates with high carbon affinity. Laser direct writing of graphene patterns on $\mathrm{Ni}$ foils has been demonstrated using methane and hydrogen gases where the number of graphene layers is controlled by the scan speed [117, 118].

A major challenge facing graphene and 2D nanosheet materials in general is cleanliness at the atomic level. Many of the applications of graphene as electrodes in organic electronics will require atomically clean surfaces to align and form well-defined interfaces with organic molecules, as shown in Fig. 7.13c [119]. Realizing such surfaces is a huge challenge, especially considering the aforementioned etching and polymer transfer processes described above. When imaged by atomic-resolution Z-contrast STEM, such as in Fig. 7.13a and b, it is seen that such processes leave large quantities of residual atoms, molecules, and polymers such that just small regions of atomically clean interface are visible. Cleaning and desorption of these adsorbates from such delicate substrates is a major challenge that lasers may be able to provide.

\subsection{Summary}

In summary, the role of laser interactions to understand and control nanomaterials synthesis has been briefly examined. Laser interactions provide unique capabilities to remotely deliver excitation to alter, diagnose, and remotely characterize the synthesis and processing of nanomaterials. 
Laser vaporization remains a special exploratory tool to create nonequilibrium growth environments that can capture metastable phases and structures of nanomaterials with novel nanoscale properties. Through the development of coordinated in situ laser spectroscopy and imaging diagnostics, the timescales for nanomaterial growth in pulsed laser plasmas and on substrates is revealed. By controlling the growth environment, and with advances in atomic resolution transmission electron microscopy, it is becoming possible to reveal the ultrasmall building blocks that serve as reactive intermediates in growth of loose nanoparticles, nanotubes, nanowires, nanohorns, and nanosheets in laser ablation plumes as well as nanorods and nanostructured thin films deposited on substrates.

Real-time understanding of the kinetics of metal-catalyst-assisted nanotube and graphene growth on substrates during chemical vapor deposition was shown as provided by laser interferometry, attenuation, and Raman scattering. Through such diagnostics, one can remotely characterize the diameter, density, alignment, and kinetics of nanomaterials in real-time. Through the introduction of pulsed gas delivery in CVD, essential nucleation and growth kinetics are revealed, providing invaluable insight into growth processes essential for advanced nanomanufacturing.

The advancements in the understanding of laser interactions for real-time synthesis and characterization are applicable not only for carbon nanomaterials, which have served as the focus for this brief review, but for the great variety of new materials, such as 2D nanosheets of other layered materials, which extend beyond graphene. New challenges for these delicate materials appear very well suited to laser processing as examples above have shown, including: laser thinning and exfoliation, laser healing of defects and methods for their controllable introduction and monitoring, and laser cleaning to produce atomically clean surfaces.

Acknowledgments Synthesis science sponsored by the Materials Sciences and Engineering Division, Office of Basic Energy Sciences, U.S. Department of Energy. Characterization science and explorations of functionality performed at the Center for Nanophase Materials Sciences, and high-resolution electron microscopy was performed in part at the Shared Research Equipment Collaborative Research Center, which are both sponsored at Oak Ridge National Laboratory by the Scientific User Facilities Division, U.S. Department of Energy.

\section{References}

1. A.P. Alivisatos, J. Phys. Chem. 100(31), 13226 (1996)

2. C.C. Chen, A.B. Herhold, C.S. Johnson, A.P. Alivisatos, Science 276(5311), 398 (1997)

3. P.F. McMillan, Nat. Mater. 1(1), 19 (2002)

4. D.B. Geohegan, in Pulsed Laser Deposition of Thin Films ed. by D.H. Chrisey, G.K. Hubler (Wiley-VCH, New York, 1994)

5. S. Amoruso, R. Bruzzese, N. Spinelli, R. Velotta, J. Phys. B-at Mol. Opt. 32(14), R131 (1999)

6. R.E. Smalley, Acc. Chem. Res. 25(3), 98 (1992)

7. S. Iijima, M. Yudasaka, R. Yamada, S. Bandow, K. Suenaga, F. Kokai, K. Takahashi, Chem. Phys. Lett. 309(3-4), 165 (1999) 
8. A.A. Puretzky, D.J. Styers-Barnett, C.M. Rouleau, H. Hu, B. Zhao, I.N. Ivanov, D.B. Geohegan, Appl. Phys. A -Mater. 93(4), 849 (2008)

9. G.W. Yang, Prog. Mater. Sci. 52(4), 648 (2007)

10. K.S. Novoselov, V.I. Fal'ko, L. Colombo, P.R. Gellert, M.G. Schwab, K. Kim, Nature 490(7419), 192 (2012)

11. A.A. Puretzky, D.B. Geohegan, S. Jesse, I.N. Ivanov, G. Eres, Appl. Phys. A-Mater. 81(2), 223 (2005)

12. A.M. Morales, C.M. Lieber, Science 279(5348), 208 (1998)

13. DOE, New Science for a Secure and Sustainable Energy Future, A Report of a Subcommittee to the Basic Energy Science Advisory Committee. (U.S. Department of Energy, Dec 2008)

14. D.B. Geohegan, Nato. Adv. Sci. Inst. Se 265, 165 (1994)

15. D.B. Geohegan, A.A. Puretzky, Appl. Surf. Sci. 96-8, 131 (1996)

16. D.H. Lowndes, D.B. Geohegan, A.A. Puretzky, D.P. Norton, C.M. Rouleau, Science 273(5277), 898 (1996)

17. R.F. Wood, K.R. Chen, J.N. Leboeuf, A.A. Puretzky, D.B. Geohegan, Phys. Rev. Lett. 79(8), 1571 (1997)

18. R.F. Wood, J.N. Leboeuf, D.B. Geohegan, A.A. Puretzky, K.R. Chen, Phys. Rev. B 58(3), 1533 (1998)

19. D.B. Geohegan, A.A. Puretzky, G. Duscher, S.J. Pennycook, Appl. Phys. Lett. 73(4), 438 (1998)

20. D.B. Geohegan, A.A. Puretzky, G. Duscher, S.J. Pennycook, Appl. Phys. Lett. 72(23), 2987 (1998)

21. R.Q. Guo, J. Nishimura, M. Matsumoto, D. Nakamura, T. Okada, Appl. Phys. A-Mater. 93(4), 843 (2008)

22. Y.B. Zel'dovich, Y.P. Raizer, Physics of Shock Waves and High-Temperature Hydrodynamic Phenomena (Academic Press, New York, 1967)

23. A.S. Barnard, L.A. Curtiss, Nano. Lett. 5(7), 1261 (2005)

24. R.L. Penn, J.F. Banfield, Science 281(5379), 969 (1998)

25. C.R.A. Catlow, S.T. Bromley, S. Hamad, M. Mora-Fonz, A.A. Sokol, S.M. Woodley, Phys. Chem. Chem. Phys. 12(4), 786 (2010)

26. D.R. Hummer, J.D. Kubicki, P.R.C. Kent, J.E. Post, P.J. Heaney, J. Phys. Chem. C 113(11), 4240 (2009)

27. Y. Zhou, K.A. Fichthorn, J. Phys. Chem. C 116(14), 8314 (2012)

28. V.N. Koparde, P.T. Cummings, J. Phys. Chem. B 109(51), 24280 (2005)

29. M. Matsui, M. Akaogi, Mol. Simul. 6, 239 (1991)

30. S. Hamad, C.R.A. Catlow, S.M. Woodley, S. Lago, J.A. Mejias, J. Phys. Chem. B 109(33), $15741(2005)$

31. H.W. Kroto, J.R. Heath, S.C. Obrien, R.F. Curl, R.E. Smalley, Nature 318(6042), 162 (1985)

32. T.G. Dietz, M.A. Duncan, D.E. Powers, R.E. Smalley, J. Chem. Phys. 74(11), 6511 (1981)

33. Y. Yamaguchi, S. Maruyama, Chem. Phys. Lett. 286(3-4), 336 (1998)

34. B.I. Dunlap, Int. J. Quantum Chem. 64(2), 193 (1997)

35. S. Irle, G.S. Zheng, Z. Wang, K. Morokuma, J. Phys. Chem. B 110(30), 14531 (2006)

36. D.B. Geohegan, A. Puretzky, C.M. Rouleau, J.J. Jackson, G. Eres, Z. Liu, D. Styers-Barnett, H. Hu, B. Zhao, I. Ivanov, K.L. More, in Laser-Surface Interactions for New Materials Production, vol. 130, ed. by A. Miotello, P.M. Ossi (Springer, Berlin Heidelberg, 2010), p. 1

37. A.A. Puretzky, D.B. Geohegan, X. Fan, S.J. Pennycook, Appl. Phys. A-Mater. 70(2), 153 (2000)

38. A.A. Puretzky, H. Schittenhelm, X.D. Fan, M.J. Lance, L.F. Allard, D.B. Geohegan, Phys. Rev. B 65, 24 (2002)

39. F. Kokai, K. Takahashi, D. Kasuya, M. Yudasaka, S. Iijima, Appl. Surf. Sci. 197, 650 (2002) 
40. D. Kasuya, M. Yudasaka, K. Takahashi, F. Kokai, S. Iijima, J. Phys. Chem. B 106(19), 4947 (2002)

41. M.D. Cheng, D.W. Lee, B. Zhao, H. Hu, D.J. Styers-Barnett, A.A. Puretzky, D.W. DePaoli, D.B. Geohegan, E.A. Ford, P. Angelini, Nanotechnology 18(18), 185604 (2007)

42. D.B. Geohegan, A.A. Puretzky, D. Styers-Barnett, H. Hu, B. Zhao, H. Cui, C.M. Rouleau, G. Eres, J.J. Jackson, R.F. Wood, S. Pannala, J.C. Wells, Phys. Status Solidi B 244(11), 3944 (2007)

43. O.L. Krivanek, M.F. Chisholm, V. Nicolosi, T.J. Pennycook, G.J. Corbin, N. Dellby, M.F. Murfitt, C.S. Own, Z.S. Szilagyi, M.P. Oxley, S.T. Pantelides, S.J. Pennycook, Nature 464(7288), 571 (2010)

44. D.B. Geohegan, H. Schittenhelm, X. Fan, S.J. Pennycook, A.A. Puretzky, M.A. Guillorn, D.A. Blom, D.C. Joy, Appl. Phys. Lett. 78(21), 3307 (2001)

45. P.J.F. Harris, Carbon 45(2), 229 (2007)

46. R. Sen, S. Suzuki, H. Kataura, Y. Achiba, Chem. Phys. Lett. 349(5-6), 383 (2001)

47. K.R.S. Chandrakumar, J.D. Readle, C. Rouleau, A. Puretzky, D.B. Geohegan, K. More, V. Krishnan, M. Tian, G. Duscher, B. Sumpter, Nanoscale 5(5), 1849 (2013)

48. Y. Liu, C.M. Brown, D.A. Neumann, D.B. Geohegan, A.A. Puretzky, C.M. Rouleau, H. Hu, D. Styers-Barnett, P.O. Krasnov, B.I. Yakobson, Carbon 50(13), 4953 (2012)

49. A. Izadi-Najafabadi, T. Yamada, D.N. Futaba, M. Yudasaka, H. Takagi, H. Hatori, S. Iijima, K. Hata, ACS Nano 5(2), 811 (2011)

50. S.K. Doorn, L.X. Zheng, M.J. O’Connell, Y.T. Zhu, S.M. Huang, J. Liu, J. Phys. Chem. B 109(9), 3751 (2005)

51. S. Hofmann, R. Sharma, C. Ducati, G. Du, C. Mattevi, C. Cepek, M. Cantoro, S. Pisana, A. Parvez, F. Cervantes-Sodi, A.C. Ferrari, R. Dunin-Borkowski, S. Lizzit, L. Petaccia, A. Goldoni, J. Robertson, Nano Lett. 7(3), 602 (2007)

52. A. Jorio, R. Saito, J.H. Hafner, C.M. Lieber, M. Hunter, T. McClure, G. Dresselhaus, M.S. Dresselhaus, Phys. Rev. Lett. 86(6), 1118 (2001)

53. M.S. Dresselhaus, G. Dresselhaus, A. Jorio, A.G. Souza, R. Saito, Carbon 40(12), 2043 (2002)

54. R. Rao, D. Liptak, T. Cherukuri, B.I. Yakobson, B. Maruyama, Nat. Mater. 11(3), 213 (2012)

55. F. Ding, A.R. Harutyunyan, B.I. Yakobson, P Natl. Acad. Sci. USA 106(8), 2506 (2009)

56. A. Modi, N. Koratkar, E. Lass, B.Q. Wei, P.M. Ajayan, Nature 424(6945), 171 (2003)

57. J. Robertson, G. Zhong, H. Telg, C. Thomsen, J.H. Warner, G.A.D. Briggs, U. DettlaffWeglikowska, S. Roth, Appl. Phys. Lett. 93(16), 163111 (2008)

58. H. Huang, C.H. Liu, Y. Wu, S.S. Fan, Adv. Mater. 17(13), 1652 (2005)

59. I. Ivanov, A. Puretzky, G. Eres, H. Wang, Z.W. Pan, H.T. Cui, R.Y. Jin, J. Howe, D.B. Geohegan, Appl. Phys. Lett. 89(22), 223110 (2006)

60. C.L. Pint, Y.Q. Xu, M. Pasquali, R.H. Hauge, ACS Nano 2(9), 1871 (2008)

61. K. Hata, D.N. Futaba, K. Mizuno, T. Namai, M. Yumura, S. Iijima, Science 306(9), 1362 (2004)

62. S. Esconjauregui, M. Fouquet, B.C. Bayer, C. Ducati, R. Smajda, S. Hofmann, J. Robertson, ACS Nano 4(12), 7431 (2010)

63. D.B. Geohegan, A.A. Puretzky, I.N. Ivanov, S. Jesse, G. Eres, J.Y. Howe, Appl. Phys. Lett. 83(9), 1851 (2003)

64. S. Maruyama, Y. Miyauchi, Y. Murakami, S. Chiashi, New J. Phys. 5, 1 (2003)

65. J.J. Jackson, A.A. Puretzky, K.L. More, C.M. Rouleau, G. Eres, D.B. Geohegan, ACS Nano 4(12), 7573 (2010)

66. D.B. Geohegan, A.A. Puretzky, J.J. Jackson, C.M. Rouleau, G. Eres, K.L. More, ACS Nano 5(10), 8311 (2011)

67. A.A. Puretzky, D.B. Geohegan, J.J. Jackson, S. Pannala, G. Eres, C.M. Rouleau, K.L. More, N. Thonnard, J.D. Readle, Small 8(10), 1534 (2012)

68. P.B. Amama, C.L. Pint, L. McJilton, S.M. Kim, E.A. Stach, P.T. Murray, R.H. Hauge, B. Maruyama, Nano Lett. 9(1), 44 (2009) 
69. S.M. Kim, C.L. Pint, P.B. Amama, R.H. Hauge, B. Maruyama, E.A. Stach, J. Mater. Res. 25(10), 1875 (2010)

70. A.R. Harutyunyan, G.G. Chen, T.M. Paronyan, E.M. Pigos, O.A. Kuznetsov, K. Hewaparakrama, S.M. Kim, D. Zakharov, E.A. Stach, G.U. Sumanasekera, Science 326(5949), 116 (2009)

71. R.T.K. Baker, M.A. Barber, R.J. Waite, P.S. Harris, F.S. Feates, J. Catal. 26(1), 51 (1972)

72. M. Lin, J.P.Y. Tan, C. Boothroyd, K.P. Loh, E.S. Tok, Y.L. Foo, Nano Lett. 6(3), 449 (2006)

73. A. Li-Pook-Than, J. Lefebvre, P. Finnie, J. Phys. Chem. C 114(25), 11018 (2010)

74. G. Eres, A.A. Kinkhabwala, H.T. Cui, D.B. Geohegan, A.A. Puretzky, D.H. Lowndes, J. Phys. Chem. B 109(35), 16684 (2005)

75. G. Eres, C.M. Rouleau, M. Yoon, A.A. Puretzky, J.J. Jackson, D.B. Geohegan, J. Phys. Chem. C 113(35), 15484 (2009)

76. N. Latorre, E. Romeo, F. Cazana, T. Ubieto, C. Royo, J.J. Villacampa, A. Monzon, J. Phys. Chem. C 114(11), 4773 (2010)

77. A.A. Puretzky, G. Eres, C.M. Rouleau, I.N. Ivanov, D.B. Geohegan, Nanotechnology 19, 5 (2008)

78. E.R. Meshot, A.J. Hart, Appl Phys Lett 92, 11 (2008)

79. P. Vinten, P. Marshall, J. Lefebvre, P. Finnie, Nanotechnology 21, 3 (2010)

80. M. Bedewy, E.R. Meshot, H.C. Guo, E.A. Verploegen, W. Lu, A.J. Hart, J. Phys. Chem. C 113(48), 20576 (2009)

81. M. Stadermann, S.P. Sherlock, J.B. In, F. Fornasiero, H.G. Park, A.B. Artyukhin, Y.M. Wang, J.J. De Yoreo, C.P. Grigoropoulos, O. Bakajin, A.A. Chernov, A. Noy, Nano Lett. 9(2), 738 (2009)

82. Y.H. Lee, X.Q. Zhang, W.J. Zhang, M.T. Chang, C.T. Lin, K.D. Chang, Y.C. Yu, J.T.W. Wang, C.S. Chang, L.J. Li, T.W. Lin, Adv. Mater. 24(17), 2320 (2012)

83. J.V. Lauritsen, J. Kibsgaard, S. Helveg, H. Topsoe, B.S. Clausen, E. Laegsgaard, F. Besenbacher, Nat. Nanotechnol. 2(1), 53 (2007)

84. H. Wang, L.L. Yu, Y.H. Lee, Y.M. Shi, A. Hsu, M.L. Chin, L.J. Li, M. Dubey, J. Kong, T. Palacios, Nano Lett. 12(9), 4674 (2012)

85. Y.J. Zhan, Z. Liu, S. Najmaei, P.M. Ajayan, J. Lou, Small 8(7), 966 (2012)

86. K.H. Lee, H.J. Shin, J. Lee, I.Y. Lee, G.H. Kim, J.Y. Choi, S.W. Kim, Nano Lett. 12(2), 714 (2012)

87. R.Z. Ma, T. Sasaki, Adv. Mater. 22(45), 5082 (2010)

88. K.S. Novoselov, D. Jiang, F. Schedin, T.J. Booth, V.V. Khotkevich, S.V. Morozov, A.K. Geim, P Natl. Acad. Sci. USA 102(30), 10451 (2005)

89. P.A. Hu, Z.Z. Wen, L.F. Wang, P.H. Tan, K. Xiao, ACS Nano 6(7), 5988 (2012)

90. K.S. Novoselov, A.K. Geim, S.V. Morozov, D. Jiang, Y. Zhang, S.V. Dubonos, I.V. Grigorieva, A.A. Firsov, Science 306(5696), 666 (2004)

91. J.N. Coleman, M. Lotya, A. O’Neill, S.D. Bergin, P.J. King, U. Khan, K. Young, A. Gaucher, S. De, R.J. Smith, I.V. Shvets, S.K. Arora, G. Stanton, H.Y. Kim, K. Lee, G.T. Kim, G.S. Duesberg, T. Hallam, J.J. Boland, J.J. Wang, J.F. Donegan, J.C. Grunlan, G. Moriarty, A. Shmeliov, R.J. Nicholls, J.M. Perkins, E.M. Grieveson, K. Theuwissen, D.W. McComb, P.D. Nellist, V. Nicolosi, Science 331(6017), 568 (2011)

92. M. Qian, Y.S. Zhou, Y. Gao, T. Feng, Z. Sun, L. Jiang, Y.F. Lu, Appl. Surf. Sci. 258(22), $9092(2012)$

93. J.J. Hu, J.S. Zabinski, J.H. Sanders, J.E. Bultman, A.A. Voevodin, J. Phys. Chem. B 110(18), 8914 (2006)

94. Y. Miyamoto, H. Zhang, D. Tomanek, Phys. Rev. Lett. 104, 20 (2010)

95. W.S. Hummers, R.E. Offeman, J. Am. Chem. Soc. 80(6), 1339 (1958)

96. M.J. McAllister, J.L. Li, D.H. Adamson, H.C. Schniepp, A.A. Abdala, J. Liu, M. HerreraAlonso, D.L. Milius, R. Car, R.K. Prud'homme, I.A. Aksay, Chem. Mater. 19(18), 4396 (2007) 
97. S. Stankovich, D.A. Dikin, R.D. Piner, K.A. Kohlhaas, A. Kleinhammes, Y. Jia, Y. Wu, S.T. Nguyen, R.S. Ruoff, Carbon 45(7), 1558 (2007)

98. K.A. Mkhoyan, A.W. Contryman, J. Silcox, D.A. Stewart, G. Eda, C. Mattevi, S. Miller, M. Chhowalla, Nano Lett. 9(3), 1058 (2009)

99. D.A. Sokolov, K.R. Shepperd, T.M. Orlando, J. Phys. Chem. Lett. 1(18), 2633 (2010)

100. D.A. Sokolov, C.M. Rouleau, D.B. Geohegan, T.M. Orlando, Carbon 53, 9 (2013)

101. A. Castellanos-Gomez, M. Barkelid, A.M. Goossens, V.E. Calado, H.S.J. van der Zant, G.A. Steele, Nano Lett. 12(6), 3187 (2012)

102. W.A. de Heer, arXiv:1012.1644v1 MRS Bulletin (submitted) (2010)

103. S. Lee, M.F. Toney, W. Ko, J.C. Randel, H.J. Jung, K. Munakata, J. Lu, T.H. Geballe, M.R. Beasley, R. Sinclair, H.C. Manoharan, A. Salleo, ACS Nano 4(12), 7524 (2010)

104. S.N. Yannopoulos, A. Siokou, N.K. Nasikas, V. Dracopoulos, F. Ravani, G.N. Papatheodorou, Adv. Funct. Mater. 22(1), 113 (2012)

105. S. Bhaviripudi, X.T. Jia, M.S. Dresselhaus, J. Kong, Nano Lett. 10(10), 4128 (2010)

106. J.M. Wofford, S. Nie, K.F. McCarty, N.C. Bartelt, O.D. Dubon, Nano Lett. 10(12), 4890 (2010)

107. X.S. Li, Y.W. Zhu, W.W. Cai, M. Borysiak, B.Y. Han, D. Chen, R.D. Piner, L. Colombo, R.S. Ruoff, Nano Lett. 9(12), 4359 (2009)

108. S. Bae, H. Kim, Y. Lee, X.F. Xu, J.S. Park, Y. Zheng, J. Balakrishnan, T. Lei, H.R. Kim, Y.I. Song, Y.J. Kim, K.S. Kim, B. Ozyilmaz, J.H. Ahn, B.H. Hong, S. Iijima, Nat. Nanotechnol. 5(8), 574 (2010)

109. R.S. Weatherup, B.C. Bayer, R. Blume, C. Ducati, C. Baehtz, R. Schlogl, S. Hofmann, Nano Lett. 11(10), 4154 (2011)

110. X.S. Li, W.W. Cai, L. Colombo, R.S. Ruoff, Nano Lett. 9(12), 4268 (2009)

111. A. Reina, X.T. Jia, J. Ho, D. Nezich, H.B. Son, V. Bulovic, M.S. Dresselhaus, J. Kong, Nano Lett. 9(1), 30 (2009)

112. A. Gruneis, K. Kummer, D.V. Vyalikh, New J Phys 11(7), 073050 (2009)

113. K.L. Saenger, J.C. Tsang, A.A. Bol, J.O. Chu, A. Grill, C. Lavoie, Appl. Phys. Lett. 96, 15 (2010)

114. S. Chiashi, Y. Murakami, Y. Miyauchi, S. Maruyama, Chem. Phys. Lett. 386(1-3), 89 (2004)

115. A.A. Puretzky, D.B. Geohegan, S. Pannala, C.M. Rouleau, M. Regmi, N. Thonnard, G. Eres, Nanoscale. 5, 6507 (2013).

116. A.C. Ferrari, J.C. Meyer, V. Scardaci, C. Casiraghi, M. Lazzeri, F. Mauri, S. Piscanec, D. Jiang, K.S. Novoselov, S. Roth, A.K. Geim, Phys. Rev. Lett. 97, 18 (2006)

117. J.B. Park, W. Xiong, Y. Gao, M. Qian, Z.Q. Xie, M. Mitchell, Y.S. Zhou, G.H. Han, L. Jiang, Y.F. Lu, Appl. Phys. Lett. 98, 12 (2011)

118. J.B. Park, W. Xiong, Z.Q. Xie, Y. Gao, M. Qian, M. Mitchell, M. Mahjouri-Samani, Y.S. Zhou, L. Jiang, Y.F. Lu, Appl. Phys. Lett. 99, 5 (2011)

119. K. Xiao, W. Deng, J.K. Keum, M. Yoon, I.V. Vlassiouk, K.W. Clark, A.-P. Li, I.I. Kravchenko, G. Gu, E.A. Payzant, J. Am. Chem. Soc. 135(9), 8 (2013) 